2. To: (Receiving Organization) Distribution

5. Proj./Prog./Dept./Div.:

W -320

B. Qriginator Remerks:
3. From: (originating organization) Criticality and Shielding

6. Design Authority/ Design Agent/Cog. Engr.:

H. J. Goldberg

11A. Design Baseline Document? [] Yes [X] No

Calculation notes are used to document the originator's analysis. They are not to be used as the final or sole document for affecting changes to the authorization basis (authorize activities), to the design basis (justify facility modifications), or to a safety basis.

4. Related EDT No.:

\section{6}

7. Purchase Order No.:

\section{NA}

9. Equip./Component No.:

NA

10. System/Bidg./Facility"

241-C-106 \& 241-AY-102

12. Major Assm. Dwg. No.:

NA

13. Permit/Permit Application No.: NA

14. Required Response Date:

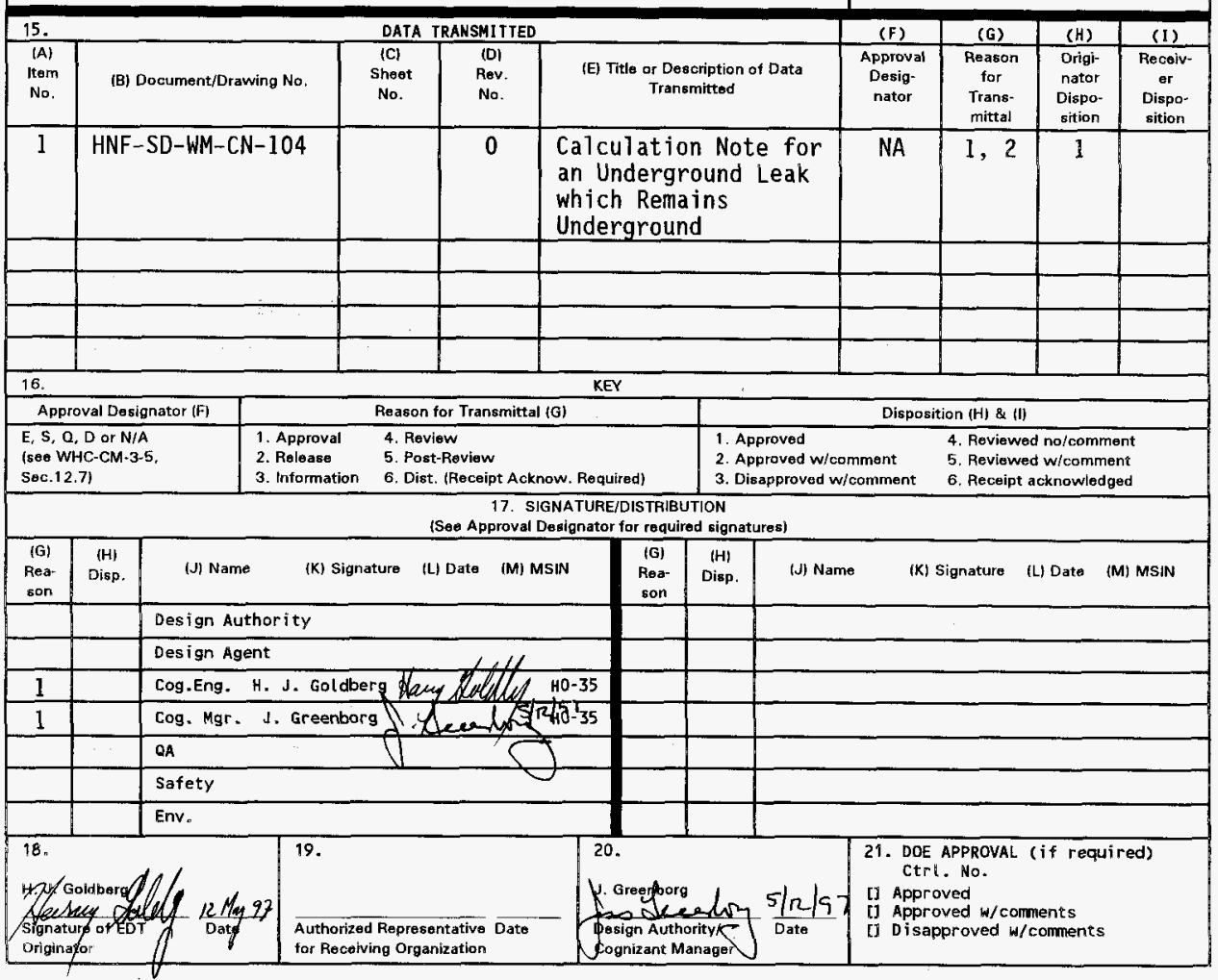

BD-7400-172-2 (05/96) GEF097 


\title{
Calculation Note for an Underground Leak which Remains Underground
}

\author{
H. J. Goldberg \\ Fluor Daniel Northwest, Inc., Richland, WA 99352 \\ U.S. Department of Energy Contract DE-AC06-96RL13200 \\ EDT/ECN: 621286 \\ Org Code: 403 \\ BRR Code: EW 3130010 \\ UC: 2030 \\ Charge Code: E09120 \\ Total Pages: 33.34
}

Key Words: Tank C-106, Waste, Pipe Leak

Abstract: The dose rate from a burst underground pipe which leaks Tank C-106 waste into the ground was calculated.

TRADEMARK DISCLAIMER. Reference herein to any specific commercial product, process, or service by trade name, trademark, manufacturer, or otherwise, does not necessarily constitute or imply its endorsement, recomendation, or favoring by the United States Government or any agency thereof or its contractors or subcontractors.

Printed in the United States of America. To obtain copies of this document, contact: Document Control Services, P.O. Box 950, Mailstop H6-08, Richland WA 99352, Phone (509) 372-2420; Fax (509) 376-4989.
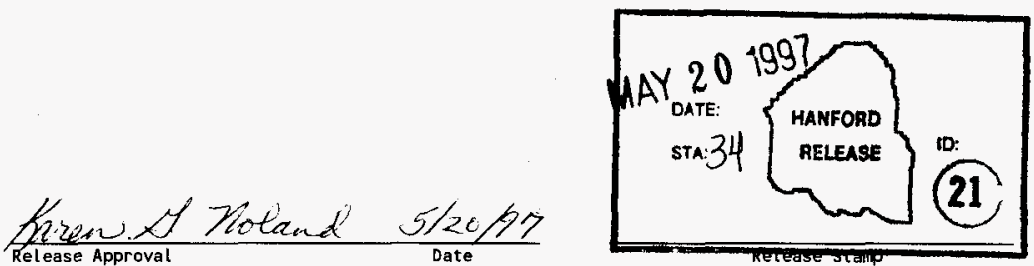

Approved for Public Release 
HNF-SD-WM-CN-104

Rev. 0

Page 1 of 33

\section{Calculation Note \\ for an \\ Underground Leak \\ which \\ Remains Underground}

This calculation note supports the subsurface leak accident scenario which remains subsurface. It is assumed that a single walled pipe carrying waste from tank 106-C ruptures, releasing the liquid waste into the soil. In this scenario, the waste does not form a surface pool, but remains subsurface. However, above the pipe is a birm, $0.762 \mathrm{~m}(2.5 \mathrm{ft}) \mathrm{high}$ and $2.44 \mathrm{~m}$ ( $8 \mathrm{ft}$ ) wide, and the liquid released from the leak rises into the birm.

The slurry line, which transports a source term of higher activity than the sluice line, leaks into the soil at a rate of $5 \%$ of the maximum flow rate of $28.4 \mathrm{~L} / \mathrm{s}(450 \mathrm{gpm})$ for twelve hours. The dose recipient was placed a perpendicular distance of $100 \mathrm{~m}$ from the pipe. Two source terms were considered, mitigated and unmitigated release as described in section 3.4.1 of HANF-SD-WM-BI0-001, Addendum 1. The unmitigated consisted of two parts of AWF Tiquid and one part AWF solid. The mitigated relese consisted of two parts SST liquid, eighteen parts AWF liquid, nine parts SST solid, and one part AWF solid. The isotopic breakdown of the release in these cases is presented in table 1 .

Two geometries were considered in preliminary investigations, disk source, and rectangular source. Since the rectangular source results from the assumption that the contamination is wicked up into the birm, only six inches of shielding from uncontaminated earth is present, while the disk source, which remains six inches below the level of the surface of the land is often shielded by a thick shield due to the slant path to the dose point. For this reason, only the rectangular source was considered in the final analysis.

The source model was a rectangle $2.134 \mathrm{~m}$ ( $7 \mathrm{ft}$ ) thick, $0.6096 \mathrm{~m}$ (2 ft) high, and $130.899 \mathrm{~m}(131 \mathrm{ft})$ long. The top and sides of this rectangular source was covered with earth of density $1.6 \mathrm{~g} / \mathrm{cm}^{3}$ to a thickness of $15.24 \mathrm{~cm}$ (6 in). This soil is modeled as $40 \%$ viod space. The source consisted of earth of the same density with the void spaces filled with the liquid waste which added $0.56 \mathrm{~g} / \mathrm{cm}^{3}$ to the density. The dose point was $100 \mathrm{~m}(328 \mathrm{ft})$ away from the birm in a perpendicular direction off the center.

The computer code MICROSKYSHINE ${ }^{\circ}$ was used to calculate the skyshine from the source. This code calculates exposure rate at the receptor point. The photon spectrum from $2 \mathrm{MeV}$ to $0.15 \mathrm{MeV}$, obtained from ISOSHLD (References 1 - 3), was used as input, although this did not differ substantially from the results obtained from using ${ }^{60} \mathrm{Co},{ }^{137 \mathrm{~m}} \mathrm{Ba}$, and ${ }^{154} \mathrm{Eu}$. However, this methodology allowed the bremsstrahlung contribution to be included in the skyshine calculation as well as in the direct rediation calculation. 
HNF-SD-WM-CN-104

Rev. 0

Page 2 of 33

\begin{tabular}{|c|c|c|c|c|}
\hline & & $10: 1.500$ & & \\
\hline \multirow[t]{2}{*}{ Isotope } & \multicolumn{2}{|c|}{ Unmitigated Release } & \multicolumn{2}{|c|}{ Mitigated Release } \\
\hline & $\mathrm{Bq}$ & $\mathrm{Ci}$ & $\mathrm{Bq}$ & $\mathrm{Ci}$ \\
\hline${ }^{60} \mathrm{Co}$ & $1.12 \mathrm{e}+13$ & $3.02 e+02$ & $9.77 e+12$ & $2.64 \mathrm{e}+02$ \\
\hline${ }^{90} \mathrm{Sr}$ & $6.61 \mathrm{e}+16$ & $1.79 e+06$ & $3.95 e+16$ & $1.07 e+06$ \\
\hline${ }^{90} y$ & $6.61 e+16$ & $1.79 \mathrm{e}+06$ & $3.95 e+16$ & $1.07 e+06$ \\
\hline${ }^{137} \mathrm{Cs}$ & $6.24 e+15$ & $1.69 \mathrm{e}+05$ & $5.98 e+15$ & $1.62 \mathrm{e}+05$ \\
\hline${ }^{137 m} \mathrm{Ba}$ & $5.90 e+15$ & $1.60 \mathrm{e}+05$ & $5.66 e+15$ & $1.53 e+05$ \\
\hline${ }^{154} \mathrm{Eu}$ & $2.50 \mathrm{e}+14$ & $6.75 e+03$ & $1.54 \mathrm{e}+14$ & $4.17 e+03$ \\
\hline${ }^{237} \mathrm{~Np}$ & $2.25 e+13$ & $6.08 e+02$ & $2.86 e+12$ & $7.74 \mathrm{e}+01$ \\
\hline${ }^{238} \mathrm{Pu}$ & $1.52 \mathrm{e}+12$ & $4.11 \mathrm{e}+01$ & $4.03 e+12$ & $1.09 \mathrm{e}+02$ \\
\hline${ }^{239} \mathrm{Pu}$ & $1.00 \mathrm{e}+13$ & $2.71 \mathrm{e}+02$ & $1.02 \mathrm{e}+13$ & $2.76 \mathrm{e}+02$ \\
\hline${ }^{241} \mathrm{Pu}$ & $3.86 \mathrm{e}+13$ & $1.04 \mathrm{e}+03$ & $7.04 e+13$ & $1.90 \mathrm{e}+03$ \\
\hline${ }^{241} \mathrm{Am}$ & $2.50 \mathrm{e}+14$ & $6.75 e+03$ & $2.99 \mathrm{e}+13$ & $8.08 \mathrm{e}+02$ \\
\hline${ }^{244} \mathrm{Cm}$ & $1.39 \mathrm{e}+12$ & $3.74 \mathrm{e}+01$ & $1.88 \mathrm{e}+11$ & $5.08 \mathrm{e}+00$ \\
\hline
\end{tabular}

The computer code ISOSHLD, which includes the contribution to the photon spectrum from bremsstrahlung, was used to calculate the direct contribution to the exposure rate. Because MICROSKYSHINE ${ }^{\circ}$ calculated only exposure rate, the exposure rate results from ISOSHLD are reported. The exposure rates for the two cases are presented in Table 2.

The skyshine is less than, but comparable to, the direct radiation in this scenario. The mitigation scenario reduces the exposure by $10 \%$, which is probably within the precision of this analysis. If we assume that one Roentgen equals one rad (which, for photons, equals one rem), the dose rate in $\mathrm{rem} / \mathrm{hr}$ can be read directly off table 2 . 


$$
\begin{array}{r}
\text { HNF-SD-WM-CN-104 } \\
\text { Rev. } 0 \\
\text { Page } 3 \text { of } 33
\end{array}
$$

\begin{tabular}{|c|c|c|c|c|}
\hline & \multicolumn{2}{|c|}{ Unmitigated Release } & \multicolumn{2}{|c|}{ Mitigated Release } \\
\hline & Roentgen/hr & Amp $/ \mathrm{kg}$ & Roentgen/hr & Amp $/ \mathrm{kg}$ \\
\hline Direct & $6.56 \mathrm{e}-02$ & $4.70 e-09$ & $5.90 e-02$ & $4.23 e-09$ \\
\hline Skyshine & $2.47 e-02$ & $1.77 e-09$ & $2.21 \mathrm{e}-02$ & $1.58 \mathrm{e}-09$ \\
\hline Total & $9.03 e-02$ & $6.47 \mathrm{e}-09$ & $8.11 \mathrm{e}-02$ & $5.81 \mathrm{e}-09$ \\
\hline
\end{tabular}

\section{References}

1) ISOSHLD- A Computer Code for General Purpose Isotope Shielding Analysis, BNWL-236, R. L. Enge1, J. Greenborg, M. M. Hendrickson, dated June 1966.

2) ISOSHLD-II: Code Revision to Include Calculation of Dose Rate from Shielded Bremsstrahlung Sources, G. L. Simmons, J. J. Regimbal, J. Greenborg, E. L. Kelly, Jr., H. H. van Tuy1, dated March 1967.

3) ISO-PC Version 1.98 - User's Guide, WHC-SD-WM-UM-030, Rev 0, P. D. Rittmann, dated May 1995. 
HNF-SD-WM-CN-104

Rev. 0

Page 4 of 33

\section{CHECKLIST FOR INDEPENDENT TECHNICAL REVIEW}

\section{DOCUMENT REVIEWED}

NUMBER: HNF-SD-WM-CN-104

TITLE: Calculation Note for an Underground Leak which Remains Underground AUTHOR(s): H. J. Goldberg

I. Method(s) of Review

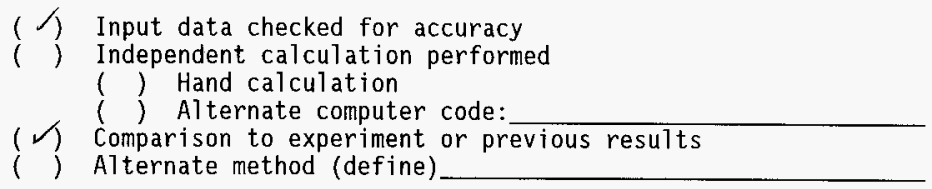

II. Checklist (either check or enter NA if not applied)

( $V$ ) Task completely defined

( $)$ Activity consistent with task specification

( $\mathscr{X}$ Necessary assumptions explicitly stated and supported

( I) Resources properly identified and referenced

(D) Resource documentation appropriate for this application

$(\checkmark)$ Input data explicitly stated

(7) Input data verified to be consistent with original source

( Geometric model adequate representation of actual geometry

$(\sqrt{ })$ Material properties appropriate and reasonable

$(\checkmark)$ Mathematical derivations checked including dimensional consistency

NA ( Hand calculations checked for errors

( $)$ Assumptions explicitly stated and justified

$(\checkmark)$ Computer software appropriate for task and used within range of validity

WA( Use of resource outside range of established validity is justified

( ) Software runstreams correct and consistent with results

( $)$ Software output consistent with input

$(C)$ Results consistent with applicable previous experimental or analytical findings

$(\mathscr{C})$ Results and conclusions address all points and are consistent with task requirements and/or established 1 imits or criteria

$N A($ ) Conclusions consistent with analytical results and established limits

$N A$ ( ) Uncertainty assesment appropriate and reasonable

( ) Other (define)

III. Comments:

IV. REVIEWER: Prate P. Kater

DATE :

$5 / 12 / 97$ 


\section{HNF-SD-WM-CN-104 \\ Rev. 0 \\ Page 5 of 33}

\section{Computer Output Deck}

Run started at $13: 57: 26 \quad 05 / 05 / 97$

ISO-PC Version 1.98

August 1994

originally ISOSHLD-II; RIBD was removed

Please send questions or comments to:

Paul D. Rittmann, PhD CHP 509-376-8715

Westinghouse Hanford Company H0-36

P0 Box $1970 \quad$ Richland, WA 99352

Title Line from Library File (ISO-PC.LIB):

Attenuation \& Buildup for 30 Groups; Photon \& Beta Production 7/6/94 PDR

Run Title: PIPE LEAK INTO BIRM-TWELVE HOUR RELEASE 
HNF-SD-WM-CN-104

Rev. 0

Page 6 of 33

UNMITIGATED 12 HOUR RELEASE - 100 M AWAY

Table of Source Activity:

Scale Factor $=1.000 \mathrm{E}+00$

\begin{tabular}{|c|c|c|}
\hline $\begin{array}{l}\text { Isotope } \\
\text { Name }\end{array}$ & $\begin{array}{l}\text { Initial } \\
\text { Values }\end{array}$ & $\begin{array}{l}\text { Final } \\
\text { Curies }\end{array}$ \\
\hline $\begin{array}{l}C O-60 \\
\text { SR- } 90 \\
Y-90 \\
C S-137 \\
\text { BA-137M } \\
\text { EU-154 } \\
\text { NP-237 } \\
\text { PU-238 } \\
P U-239 \\
P U-241 \\
A M-241 \\
C M-244\end{array}$ & $\begin{array}{l}3.02 \mathrm{E}+02 \\
1.79 \mathrm{E}+06 \\
1.79 \mathrm{E}+06 \\
1.69 \mathrm{E}+05 \\
1.60 \mathrm{E}+05 \\
6.75 \mathrm{E}+03 \\
6.08 \mathrm{E}+02 \\
4.11 \mathrm{E}+01 \\
2.71 \mathrm{E}+02 \\
1.04 \mathrm{E}+03 \\
6.75 \mathrm{E}+03 \\
3.74 \mathrm{E}+01\end{array}$ & $\begin{array}{l}3.020 \mathrm{E}+02 \\
1.790 \mathrm{E}+06 \\
1.790 \mathrm{E}+06 \\
1.690 \mathrm{E}+05 \\
1.600 \mathrm{E}+05 \\
6.750 \mathrm{E}+03 \\
6.080 \mathrm{E}+02 \\
4.110 \mathrm{E}+01 \\
2.710 \mathrm{E}+02 \\
1.040 \mathrm{E}+03 \\
6.750 \mathrm{E}+03 \\
3.740 \mathrm{E}+01\end{array}$ \\
\hline
\end{tabular}


HNF-SD-WM-CN-104

Rev. 0

Page 7 of 33

UNMITIGATED 12 HOUR RELEASE - 100 M AWAY

Shield Composition, $\mathrm{g} / \mathrm{cc}$

Shield 1 Shield 2 Shield 3

\begin{tabular}{llll} 
WATER & $5.600 \mathrm{E}-01$ & $0.000 \mathrm{E}+00$ & $0.000 \mathrm{E}+00$ \\
AIR & $0.000 \mathrm{E}+00$ & $0.000 \mathrm{E}+00$ & $1.290 \mathrm{E}-03$ \\
HAN S0IL & $1.600 \mathrm{E}+00$ & $1.600 \mathrm{E}+00$ & $0.000 \mathrm{E}+00$ \\
\hdashline Totals: & $2.160 \mathrm{E}+00$ & $1.600 \mathrm{E}+00$ & $1.290 \mathrm{E}-03$
\end{tabular}

$\mathrm{E}, \mathrm{MeV}$ Linear Attenuation, per $\mathrm{cm}$ (last region is air)

$\begin{array}{rrrr}0.015 & 2.044 \mathrm{E}+01 & 1.952 \mathrm{E}+01 & 2.046 \mathrm{E}-03 \\ 0.025 & 4.813 \mathrm{E}+00 & 4.535 \mathrm{E}+00 & 6.138 \mathrm{E}-04 \\ 0.035 & 1.986 \mathrm{E}+00 & 1.817 \mathrm{E}+00 & 3.664 \mathrm{E}-04 \\ 0.045 & 1.119 \mathrm{E}+00 & 9.853 \mathrm{E}-01 & 2.837 \mathrm{E}-04 \\ 0.055 & 7.679 \mathrm{E}-01 & 6.493 \mathrm{E}-01 & 2.491 \mathrm{E}-04 \\ 0.065 & 5.980 \mathrm{E}-01 & 4.872 \mathrm{E}-01 & 2.314 \mathrm{E}-04 \\ 0.075 & 5.029 \mathrm{E}-01 & 3.981 \mathrm{E}-01 & 2.184 \mathrm{E}-04 \\ 0.085 & 4.446 \mathrm{E}-01 & 3.442 \mathrm{E}-01 & 2.090 \mathrm{E}-04 \\ 0.095 & 4.062 \mathrm{E}-01 & 3.091 \mathrm{E}-01 & 2.019 \mathrm{E}-04 \\ 0.150 & 3.135 \mathrm{E}-01 & 2.288 \mathrm{E}-01 & 1.758 \mathrm{E}-04 \\ 0.250 & 2.546 \mathrm{E}-01 & 1.835 \mathrm{E}-01 & 1.477 \mathrm{E}-04 \\ 0.350 & 2.229 \mathrm{E}-01 & 1.603 \mathrm{E}-01 & 1.298 \mathrm{E}-04 \\ 0.475 & 1.961 \mathrm{E}-01 & 1.408 \mathrm{E}-01 & 1.146 \mathrm{E}-04 \\ 0.650 & 1.710 \mathrm{E}-01 & 1.227 \mathrm{E}-01 & 1.001 \mathrm{E}-04 \\ 0.825 & 1.533 \mathrm{E}-01 & 1.099 \mathrm{E}-01 & 8.984 \mathrm{E}-05 \\ 1.000 & 1.397 \mathrm{E}-01 & 1.002 \mathrm{E}-01 & 8.194 \mathrm{E}-05 \\ 1.225 & 1.263 \mathrm{E}-01 & 9.058 \mathrm{E}-02 & 7.398 \mathrm{E}-05 \\ 1.475 & 1.150 \mathrm{E}-01 & 8.246 \mathrm{E}-02 & 6.718 \mathrm{E}-05 \\ 1.700 & 1.068 \mathrm{E}-01 & 7.669 \mathrm{E}-02 & 6.249 \mathrm{E}-05 \\ 1.900 & 1.008 \mathrm{E}-01 & 7.243 \mathrm{E}-02 & 5.889 \mathrm{E}-05 \\ 2.100 & 9.574 \mathrm{E}-02 & 6.882 \mathrm{E}-02 & 5.564 \mathrm{E}-05 \\ 2.300 & 9.133 \mathrm{E}-02 & 6.573 \mathrm{E}-02 & 5.263 \mathrm{E}-05 \\ 2.500 & 8.750 \mathrm{E}-02 & 6.304 \mathrm{E}-02 & 5.003 \mathrm{E}-05 \\ 2.700 & 8.418 \mathrm{E}-02 & 6.072 \mathrm{E}-02 & 4.778 \mathrm{E}-05 \\ 3.000 & 7.995 \mathrm{E}-02 & 5.776 \mathrm{E}-02 & 4.511 \mathrm{E}-05 \\ 3.600 & 7.338 \mathrm{E}-02 & 5.323 \mathrm{E}-02 & 4.158 \mathrm{E}-05 \\ 4.500 & 6.631 \mathrm{E}-02 & 4.842 \mathrm{E}-02 & 3.746 \mathrm{E}-05 \\ 5.500 & 6.089 \mathrm{E}-02 & 4.474 \mathrm{E}-02 & 3.381 \mathrm{E}-05 \\ 6.600 & 5.677 \mathrm{E}-02 & 4.198 \mathrm{E}-02 & 3.111 \mathrm{E}-05 \\ 8.600 & 5.210 \mathrm{E}-02 & 3.898 \mathrm{E}-02 & 2.771 \mathrm{E}-05 \\ ---------------0 & \end{array}$




\section{HNF-SD-WM-CN-104 \\ Rev. 0 \\ Page 8 of 33}

\section{UNMITIGATED 12 HOUR RELEASE - 100 M AWAY}

Photon Production Rate for Each Radionuclide:

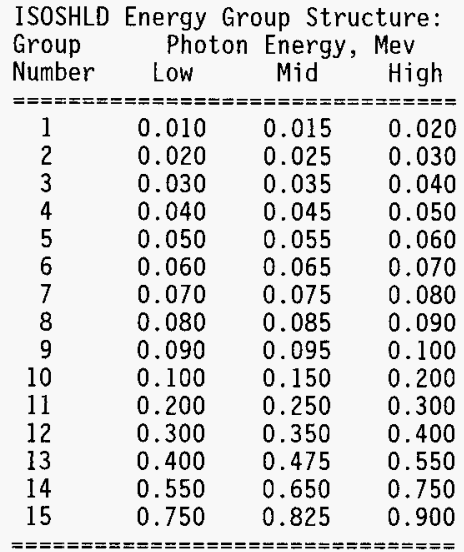

\begin{tabular}{|c|c|c|c|}
\hline $\begin{array}{l}\text { Nucl i } \\
\text { Group } \\
\text { No. }\end{array}$ & $\begin{array}{l}472 \text { is } \\
\text { Gamma } \\
X-r a y\end{array}$ & $\begin{array}{l}\mathrm{CO}-60 \\
\text { Brems }\end{array}$ & $\begin{array}{c}(Z=27) \\
\text { Total } \\
\text { photon/sec }\end{array}$ \\
\hline $\begin{array}{rl}1 & 0 \\
2 & 0 \\
3 & 0 \\
4 & 0 \\
5 & 0 \\
6 & 0 \\
7 & 0 \\
8 & 0 \\
9 & 0 \\
10 & 0 \\
11 & 0 \\
12 & 0 \\
15 & 1\end{array}$ & $\begin{array}{l}000 \mathrm{E}+00 \\
000 \mathrm{E}+00 \\
000 \mathrm{E}+00 \\
000 \mathrm{E}+00 \\
000 \mathrm{E}+00 \\
000 \mathrm{E}+00 \\
000 \mathrm{E}+00 \\
000 \mathrm{E}+00 \\
000 \mathrm{E}+00 \\
000 \mathrm{E}+00 \\
000 \mathrm{E}+00 \\
000 \mathrm{E}+00 \\
117 \mathrm{E}+09\end{array}$ & $\begin{array}{l}2.863 \mathrm{E}+10 \\
9.936 \mathrm{E}+09 \\
5.395 \mathrm{E}+09 \\
3.180 \mathrm{E}+09 \\
2.055 \mathrm{E}+09 \\
1.432 \mathrm{E}+09 \\
9.910 \mathrm{E}+08 \\
7.088 \mathrm{E}+08 \\
5.151 \mathrm{E}+08 \\
1.350 \mathrm{E}+09 \\
3.705 \mathrm{E}+07 \\
1.555 \mathrm{E}+04 \\
0.000 \mathrm{E}+00\end{array}$ & $\begin{array}{l}2.863 \mathrm{E}+10 \\
9.936 \mathrm{E}+09 \\
5.395 \mathrm{E}+09 \\
3.180 \mathrm{E}+09 \\
2.055 \mathrm{E}+09 \\
1.432 \mathrm{E}+09 \\
9.910 \mathrm{E}+08 \\
7.088 \mathrm{E}+08 \\
5.151 \mathrm{E}+08 \\
1.350 \mathrm{E}+09 \\
3.705 \mathrm{E}+07 \\
1.555 \mathrm{E}+04 \\
1.117 \mathrm{E}+09\end{array}$ \\
\hline
\end{tabular}

$\begin{array}{cccc}\begin{array}{c}\text { Group } \\ \text { Number } \\ ========\end{array} & \begin{array}{c}\text { Photon } \\ \text { Low }\end{array} & \begin{array}{c}\text { Energy, } \\ \text { Mid }\end{array} & \begin{array}{c}\text { Mev } \\ \text { High }\end{array} \\ 16 & 0.900 & 1.000 & 1.100 \\ 17 & 1.100 & 1.225 & 1.350 \\ 18 & 1.350 & 1.475 & 1.600 \\ 19 & 1.600 & 1.700 & 1.800 \\ 20 & 1.800 & 1.900 & 2.000 \\ 21 & 2.000 & 2.100 & 2.200 \\ 22 & 2.200 & 2.300 & 2.400 \\ 23 & 2.400 & 2.500 & 2.600 \\ 24 & 2.600 & 2.700 & 2.800 \\ 25 & 2.800 & 3.000 & 3.200 \\ 26 & 3.200 & 3.600 & 4.000 \\ 27 & 4.000 & 4.500 & 5.000 \\ 28 & 5.000 & 5.500 & 6.000 \\ 29 & 6.000 & 6.600 & 7.200 \\ 30 & 7.200 & 8.600 & 10.000 \\ ============================\end{array}$

Weight (472) $=3.020 \mathrm{E}+02 \mathrm{Ci}$ Group Gamma Total No. X-ray Brems photon/sec

$16 \quad 0.000 \mathrm{E}+00 \quad 0.000 \mathrm{E}+00 \quad 0.000 \mathrm{E}+00$

$172.235 \mathrm{E}+13 \quad 0.000 \mathrm{E}+00 \quad 2.235 \mathrm{E}+13$

$180.000 \mathrm{E}+00 \quad 0.000 \mathrm{E}+00 \quad 0.000 \mathrm{E}+00$

$190.000 \mathrm{E}+00 \quad 0.000 \mathrm{E}+00 \quad 0.000 \mathrm{E}+00$

$200.000 \mathrm{E}+00 \quad 0.000 \mathrm{E}+00 \quad 0.000 \mathrm{E}+00$

$210.000 \mathrm{E}+00 \quad 0.000 \mathrm{E}+00 \quad 0.000 \mathrm{E}+00$

$22 \quad 0.000 \mathrm{E}+00 \quad 0.000 \mathrm{E}+00 \quad 0.000 \mathrm{E}+00$

$230.000 \mathrm{E}+00 \quad 0.000 \mathrm{E}+00 \quad 0.000 \mathrm{E}+00$

$240.000 \mathrm{E}+00 \quad 0.000 \mathrm{E}+00 \quad 0.000 \mathrm{E}+00$

$250.000 \mathrm{E}+00 \quad 0.000 \mathrm{E}+00 \quad 0.000 \mathrm{E}+00$

$260.000 \mathrm{E}+00 \quad 0.000 \mathrm{E}+00 \quad 0.000 \mathrm{E}+00$

$270.000 \mathrm{E}+00 \quad 0.000 \mathrm{E}+00 \quad 0.000 \mathrm{E}+00$

$30 \quad 0.000 \mathrm{E}+00 \quad 0.000 \mathrm{E}+00 \quad 0.000 \mathrm{E}+00$

Totals: $2.235 \mathrm{E}+13 \quad 5.423 \mathrm{E}+10 \quad 2.240 \mathrm{E}+13$ 
HNF-SD-WM-CN-104

Rev. 0

Page 9 of 33

\begin{tabular}{|c|c|c|c|}
\hline $\begin{array}{l}\text { Nuclio } \\
\text { aroup } \\
\text { No. }\end{array}$ & $\begin{array}{l}\text { Gamma } \\
\text { X-ray }\end{array}$ & Brems & $\begin{array}{c}(Z=38) \\
\text { Total } \\
\text { photon } / \mathrm{sec}\end{array}$ \\
\hline $\begin{array}{rl}1 & 0 . \\
2 & 0 . \\
3 & 0 . \\
4 & 0 . \\
5 & 0 . \\
6 & 0 . \\
7 & 0 . \\
8 & 0 . \\
9 & 0 . \\
10 & 0 . \\
11 & 0 . \\
12 & 0 . \\
13 & 0 .\end{array}$ & $\begin{array}{l}000 \mathrm{E}+00 \\
000 \mathrm{E}+00 \\
000 \mathrm{E}+00 \\
000 \mathrm{E}+00 \\
000 \mathrm{E}+00 \\
000 \mathrm{E}+00 \\
000 \mathrm{E}+00 \\
000 \mathrm{E}+00\end{array}$ & $\begin{array}{l}2.807 \mathrm{E}+14 \\
1.737 \mathrm{E}+14 \\
9.778 \mathrm{E}+13 \\
4.905 \mathrm{E}+13 \\
3.667 \mathrm{E}+13 \\
2.554 \mathrm{E}+13 \\
1.899 \mathrm{E}+13 \\
1.507 \mathrm{E}+13 \\
1.131 \mathrm{E}+13 \\
4.324 \mathrm{E}+13 \\
6.885 \mathrm{E}+12\end{array}$ & $\begin{array}{l}2.80 \\
1.73 \\
9.7 \\
4.90 \\
3.60 \\
2.55 \\
1.80 \\
1.50 \\
1.13 \\
4.32 \\
6.88 \\
1.01 \\
1.07\end{array}$ \\
\hline
\end{tabular}

Weight $(82)=1.790 E+06 \mathrm{Ci}$

Group Gamma Total

No. X-ray Brems photon/sec

$\begin{array}{lll}16 & 0.000 \mathrm{E}+00 & 0.000 \mathrm{E}+00 \quad 0.000 \mathrm{E}+00\end{array}$

$17 \quad 0.000 \mathrm{E}+00 \quad 0.000 \mathrm{E}+00 \quad 0.000 \mathrm{E}+00$

$18 \quad 0.000 \mathrm{E}+00 \quad 0.000 \mathrm{E}+00 \quad 0.000 \mathrm{E}+00$

$19 \quad 0.000 \mathrm{E}+00 \quad 0.000 \mathrm{E}+00 \quad 0.000 \mathrm{E}+00$

$20 \quad 0.000 \mathrm{E}+00 \quad 0.000 \mathrm{E}+00 \quad 0.000 \mathrm{E}+00$

2l $0.000 \mathrm{E}+00 \quad 0.000 \mathrm{E}+00 \quad 0.000 \mathrm{E}+00$

$22 \quad 0.000 \mathrm{E}+00 \quad 0.000 \mathrm{E}+00 \quad 0.000 \mathrm{E}+00$

$23 \quad 0.000 \mathrm{E}+00 \quad 0.000 \mathrm{E}+00 \quad 0.000 \mathrm{E}+00$

$24 \quad 0.000 \mathrm{E}+00 \quad 0.000 \mathrm{E}+00 \quad 0.000 \mathrm{E}+00$

$\begin{array}{lll}25 & 0.000 \mathrm{E}+00 \quad 0.000 \mathrm{E}+00 \quad 0.000 \mathrm{E}+00\end{array}$

$\begin{array}{llll}26 & 0.000 \mathrm{E}+00 & 0.000 \mathrm{E}+00 & 0.000 \mathrm{E}+00\end{array}$

$27 \quad 0.000 E+00 \quad 0.000 E+00 \quad 0.000 E+00$

$280.000 \mathrm{E}+00 \quad 0.000 \mathrm{E}+00 \quad 0.000 \mathrm{E}+00$

Totals: $0.000 \mathrm{E}+00 \quad 7.601 \mathrm{E}+147.601 \mathrm{E}+14$

\begin{tabular}{|c|c|c|c|c|c|c|c|}
\hline $\begin{array}{l}\text { Nucl id } \\
\text { Group } \\
\text { No. }\end{array}$ & $\begin{array}{l}\text { le } 84 \text { is } \\
\text { Gamma } \\
\text { X-ray }\end{array}$ & $\begin{array}{l}Y-90 \\
\text { Brems }\end{array}$ & $\begin{array}{c}(Z=39) \\
\text { Total } \\
\text { photon } / \mathrm{sec}\end{array}$ & $\begin{array}{l}\text { Weigl } \\
\text { Group } \\
\text { No. }\end{array}$ & $\begin{array}{l}t(84)= \\
\text { Gamma } \\
\text { X-ray }\end{array}$ & $=1.790 \mathrm{E}+06$ & $\begin{array}{l}6 \mathrm{Ci} \\
\text { Total } \\
\text { photon/sec }\end{array}$ \\
\hline $\begin{array}{rl}1 & 5 . \\
2 & 0 . \\
3 & 0 . \\
4 & 0 . \\
5 & 0 . \\
6 & 0 . \\
7 & 0 . \\
8 & 0 . \\
9 & 0 . \\
10 & 0 . \\
11 & 0 . \\
12 & 0 . \\
13 & 0 . \\
14 & 0 . \\
15 & 0 .\end{array}$ & $\begin{array}{l}033 E+12 \\
000 E+00 \\
000 E+00 \\
000 E+00 \\
000 E+00 \\
000 E+00 \\
000 E+00 \\
000 E+00 \\
000 E+00 \\
000 E+00 \\
000 F+00\end{array}$ & $\begin{array}{l}1.849 \mathrm{E}+15 \\
9.384 \mathrm{E}+14 \\
5.850 \mathrm{E}+14 \\
4.210 \mathrm{E}+14 \\
3.039 \mathrm{E}+14 \\
2.654 \mathrm{E}+14 \\
2.455 \mathrm{E}+14 \\
2.255 \mathrm{E}+14 \\
2.056 \mathrm{E}+14 \\
1.044 \mathrm{E}+15\end{array}$ & $\begin{array}{l}1.854 \mathrm{E}+15 \\
9.384 \mathrm{E}+14 \\
5.850 \mathrm{E}+14 \\
4.210 \mathrm{E}+14 \\
3.039 \mathrm{E}+14 \\
2.654 \mathrm{E}+14 \\
2.455 \mathrm{E}+14 \\
2.255 \mathrm{E}+14 \\
2.056 \mathrm{E}+14 \\
1.044 \mathrm{E}+15 \\
3.303 \mathrm{E}+14 \\
1.626 \mathrm{E}+14 \\
1.248 \mathrm{E}+14 \\
7.574 \mathrm{E}+13 \\
2.744 \mathrm{E}+13\end{array}$ & $\begin{array}{ll}16 & 0 \\
17 & 0 \\
18 & 0 \\
19 & 2 \\
20 & 0 \\
21 & 9 \\
22 & 0 \\
23 & 0 \\
24 & 0 \\
25 & 0 \\
26 & 0 \\
27 & 0 \\
28 & 0 \\
29 & 0 \\
30 & 0\end{array}$ & $\begin{array}{l}000 \mathrm{E}+00 \\
000 \mathrm{E}+00 \\
000 \mathrm{E}+00 \\
252 \mathrm{E}+12 \\
000 \mathrm{E}+00 \\
272 \mathrm{E}+10 \\
000 \mathrm{E}+00 \\
000 \mathrm{E}+00 \\
000 \mathrm{E}+00 \\
000 \mathrm{E}+00 \\
000 \mathrm{E}+00 \\
000 \mathrm{E}+00 \\
000 \mathrm{E}+00 \\
000 \mathrm{E}+00 \\
000 \mathrm{E}+00\end{array}$ & $\begin{array}{l}1.872 \mathrm{E}+13 \\
9.646 \mathrm{E}+12 \\
3.191 \mathrm{E}+12 \\
7.278 \mathrm{E}+11 \\
1.610 \mathrm{E}+11 \\
1.350 \mathrm{E}+10 \\
1.981 \mathrm{E}+08 \\
0.000 \mathrm{E}+00 \\
0.000 \mathrm{E}+00 \\
0.000 \mathrm{E}+00 \\
0.000 \mathrm{E}+00 \\
0.000 \mathrm{E}+00 \\
0.000 \mathrm{E}+00 \\
0.000 \mathrm{E}+00 \\
0.000 \mathrm{E}+00\end{array}$ & $\begin{array}{l}1.872 \mathrm{E}+13 \\
9.646 \mathrm{E}+12 \\
3.191 \mathrm{E}+12 \\
2.980 \mathrm{E}+12 \\
1.610 \mathrm{E}+11 \\
1.062 \mathrm{E}+11 \\
1.981 \mathrm{E}+08 \\
0.000 \mathrm{E}+00 \\
0.000 \mathrm{E}+00 \\
0.000 \mathrm{E}+00 \\
0.000 \mathrm{E}+00 \\
0.000 \mathrm{E}+00 \\
0.000 \mathrm{E}+00 \\
0.000 \mathrm{E}+00 \\
0.000 \mathrm{E}+00\end{array}$ \\
\hline
\end{tabular}

Totals: $7.378 \mathrm{E}+12 \quad 6.837 \mathrm{E}+15 \quad 6.844 \mathrm{E}+15$ 
HNF-SD-WM-CN-104

Rev. 0

Page 10 of 33

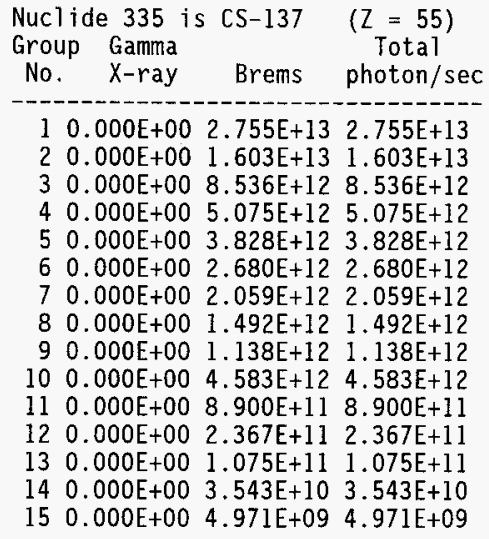

Weight $(335)=1.690 \mathrm{E}+05 \mathrm{Ci}$

Group Gamma Total

No. X-ray Brems photon/sec

$160.000 \mathrm{E}+00 \quad 7.425 \mathrm{E}+08 \quad 7.425 \mathrm{E}+08$

$170.000 \mathrm{E}+00 \quad 5.579 \mathrm{E}+06 \quad 5.579 \mathrm{E}+06$

$180.000 \mathrm{E}+00 \quad 0.000 \mathrm{E}+00 \quad 0.000 \mathrm{E}+00$

$190.000 \mathrm{E}+00 \quad 0.000 \mathrm{E}+00 \quad 0.000 \mathrm{E}+00$

$20 \quad 0.000 \mathrm{E}+00 \quad 0.000 \mathrm{E}+00 \quad 0.000 \mathrm{E}+00$

$21 \quad 0.000 \mathrm{E}+00 \quad 0.000 \mathrm{E}+00 \quad 0.000 \mathrm{E}+00$

$22 \quad 0.000 \mathrm{E}+00 \quad 0.000 \mathrm{E}+00 \quad 0.000 \mathrm{E}+00$

$23 \quad 0.000 \mathrm{E}+00 \quad 0.000 \mathrm{E}+00 \quad 0.000 \mathrm{E}+00$

$240.000 \mathrm{E}+00 \quad 0.000 \mathrm{E}+00 \quad 0.000 \mathrm{E}+00$

$250.000 \mathrm{E}+00 \quad 0.000 \mathrm{E}+00 \quad 0.000 \mathrm{E}+00$

$260.000 \mathrm{E}+00 \quad 0.000 \mathrm{E}+00 \quad 0.000 \mathrm{E}+00$

$27 \quad 0.000 \mathrm{E}+00 \quad 0.000 \mathrm{E}+00 \quad 0.000 \mathrm{E}+00$

$280.000 \mathrm{E}+00 \quad 0.000 \mathrm{E}+00 \quad 0.000 \mathrm{E}+00$

$290.000 \mathrm{E}+00 \quad 0.000 \mathrm{E}+00 \quad 0.000 \mathrm{E}+00$

$300.000 \mathrm{E}+00 \quad 0.000 \mathrm{E}+00 \quad 0.000 \mathrm{E}+00$

Totals: $0.000 \mathrm{E}+00 \quad 7.424 \mathrm{E}+13 \quad 7.424 \mathrm{E}+13$

\begin{tabular}{|c|c|c|c|c|c|c|c|}
\hline Nucl id & 336 is & BA-137M & $\left(Z_{-}=56\right)$ & Weigh & $(336)$ & $=1.600 \mathrm{E}+0$ & $5 \mathrm{Ci}$ \\
\hline $\begin{array}{l}\text { Group } \\
\text { No. }\end{array}$ & $\begin{array}{l}\text { Gamma } \\
X-r a y\end{array}$ & Brems & $\begin{array}{c}\text { Total } \\
\text { photon/sec }\end{array}$ & $\begin{array}{l}\text { Group } \\
\text { No. }\end{array}$ & $\begin{array}{l}\text { Gamma } \\
X-r a y\end{array}$ & & $\begin{array}{c}\text { Tota } 7 \\
\text { photon } / \mathrm{sec}\end{array}$ \\
\hline $\begin{array}{rr}3 & 4.3 \\
14 & 5.3\end{array}$ & $327 E+15$ & $\begin{array}{l}0.000 \mathrm{E}+00 \\
0.000 \mathrm{E}+00\end{array}$ & $\begin{array}{l}4.310 \mathrm{E}+14 \\
5.327 \mathrm{E}+15\end{array}$ & $\begin{array}{ll}180 . \\
29 & 0 .\end{array}$ & & $\begin{array}{l}0.000 \mathrm{E}+00 \\
0.000 \mathrm{E}+00\end{array}$ & $\begin{array}{l}0.000 \mathrm{E}+00 \\
0.000 \mathrm{E}+00\end{array}$ \\
\hline
\end{tabular}

Totals: $5.758 \mathrm{E}+15 \quad 0.000 \mathrm{E}+00 \quad 5.758 \mathrm{E}+15$

\begin{tabular}{|c|c|c|c|c|c|c|c|}
\hline $\begin{array}{l}\text { Nucli } \\
\text { Group } \\
\text { No. }\end{array}$ & $\begin{array}{l}415 \text { is } \\
\text { Gamma } \\
\text { X-ray }\end{array}$ & Brems & $\begin{array}{l}(Z=63) \\
\text { Total } \\
\text { photon } / \mathrm{sec}\end{array}$ & $\begin{array}{l}\text { Weigh } \\
\text { Group } \\
\text { No. }\end{array}$ & $\begin{array}{c}\text { t }(415)= \\
\text { Gamma } \\
\text { X-ray }\end{array}$ & $=6.750 \mathrm{E}+03$ & $\begin{array}{l}3 \mathrm{Cj} \\
\text { Total } \\
\text { photon/sec }\end{array}$ \\
\hline $\begin{array}{rl}1 & 0 \\
2 & 0 \\
3 & 0 \\
4 & 6 \\
5 & 0 \\
6 & 0 \\
7 & 0 \\
8 & 0 \\
9 & 0 \\
10 & 1 \\
11 & 1 \\
12 & 0 \\
13 & 2 \\
14 & 8 \\
15 & 3\end{array}$ & $\begin{array}{l}00 \\
.00 \\
-13 \\
.00 \\
.00 \\
.00 \\
.00 \\
.00 \\
14 \\
13 \\
.00 \\
12\end{array}$ & $\begin{array}{l}7.7 \\
5.0 \\
3.1 \\
2.3 \\
1.8 \\
1.5 \\
1.2 \\
1.0 \\
4.2 \\
1.2 \\
5.1 \\
3.3\end{array}$ & $\begin{array}{l}7.7 \\
5.0 \\
6.4 \\
2.3 \\
1.8 \\
1.5 \\
1.2\end{array}$ & $\begin{array}{ll}16 & 7 . \\
17 & 9 . \\
18 & 8 . \\
19 & 0 . \\
20 & 0 . \\
21 & 0 . \\
22 & 0 . \\
23 & 0 . \\
24 & 0 . \\
25 & 0 . \\
26 & 0 . \\
27 & 0 . \\
28 & 0 . \\
29 & 0 . \\
30 & 0 .\end{array}$ & & & $\begin{array}{l}7.073 \mathrm{E}+13 \\
9.268 \mathrm{E}+13 \\
8.816 \mathrm{E}+12 \\
6.873 \mathrm{E}+06 \\
6.541 \mathrm{E}+03 \\
0.000 \mathrm{E}+00 \\
0.000 \mathrm{E}+00 \\
0.000 \mathrm{E}+00 \\
0.000 \mathrm{E}+00 \\
0.000 \mathrm{E}+00 \\
0.000 \mathrm{E}+00 \\
0.000 \mathrm{E}+00 \\
0.000 \mathrm{E}+00 \\
0.000 \mathrm{E}+00 \\
0.000 \mathrm{E}+00\end{array}$ \\
\hline
\end{tabular}


HNF-SD-WM-CN-104 Rev. 0

Page 11 of 33

Totals: $4.760 \mathrm{E}+14 \quad 4.592 \mathrm{E}+12 \quad 4.806 \mathrm{E}+14$ 
HNF-SD-WM-CN-104

Rev. 0

Page 12 of 33

\begin{tabular}{|c|c|c|c|}
\hline $\begin{array}{l}\text { Nuclide } \\
\text { Group } \\
\text { No. }\end{array}$ & $\begin{array}{l}502 \text { is } \\
\text { Gamma } \\
X-r a y\end{array}$ & $N P-237$ & $\begin{array}{c}(Z=93) \\
\text { Total } \\
\text { photon/se }\end{array}$ \\
\hline $\begin{array}{rl}1 & 1 \\
2 & 3 \\
3 & 0 \\
4 & 3 \\
5 & 9 \\
6 & 0 \\
7 & 0 \\
8 & 2 \\
9 & 1 \\
10 & 7 \\
11 & 3\end{array}$ & $\begin{array}{l}332 \mathrm{E}+13 \\
147 \mathrm{E}+12 \\
000 \mathrm{E}+00 \\
149 \mathrm{E}+10 \\
448 \mathrm{E}+10 \\
000 \mathrm{E}+00 \\
000 \mathrm{E}+00 \\
870 \mathrm{E}+12 \\
125 \mathrm{E}+12 \\
424 \mathrm{E}+11\end{array}$ & $\begin{array}{l}1.330 \mathrm{E}+09 \\
2.330 \mathrm{E}+08 \\
5.441 \mathrm{E}+07 \\
1.458 \mathrm{E}+07 \\
4.404 \mathrm{E}+06 \\
1.432 \mathrm{E}+06 \\
4.801 \mathrm{E}+05 \\
1.554 \mathrm{E}+05 \\
3.832 \mathrm{E}+04 \\
1.499 \mathrm{E}+04 \\
0.000 \mathrm{E}+00\end{array}$ & $\begin{array}{l}1.332 \mathrm{E}+13 \\
3.147 \mathrm{E}+12 \\
5.441 \mathrm{E}+07 \\
3.151 \mathrm{E}+10 \\
9.449 \mathrm{E}+10 \\
1.432 \mathrm{E}+06 \\
4.801 \mathrm{E}+05 \\
2.870 \mathrm{E}+12 \\
1.125 \mathrm{E}+12 \\
7.424 \mathrm{E}+11 \\
3.599 \mathrm{E}+10\end{array}$ \\
\hline
\end{tabular}

$\begin{array}{cccc}\begin{array}{c}\text { Weight }(502)= \\ \text { Group Gamma } \\ \text { No. }\end{array} \text { X-ray } & \text { Brems } & \begin{array}{c}\text { Total } \\ \text { photon } / \mathrm{sec}\end{array} \\ -16 & 0.000 \mathrm{E}+00 & 0.000 \mathrm{E}+00 & 0.000 \mathrm{E}+00 \\ 17 & 0.000 \mathrm{E}+00 & 0.000 \mathrm{E}+00 & 0.000 \mathrm{E}+00 \\ 18 & 0.000 \mathrm{E}+00 & 0.000 \mathrm{E}+00 & 0.000 \mathrm{E}+00 \\ 19 & 0.000 \mathrm{E}+00 & 0.000 \mathrm{E}+00 & 0.000 \mathrm{E}+00 \\ 20 & 0.000 \mathrm{E}+00 & 0.000 \mathrm{E}+00 & 0.000 \mathrm{E}+00 \\ 21 & 0.000 \mathrm{E}+00 & 0.000 \mathrm{E}+00 & 0.000 \mathrm{E}+00 \\ 22 & 0.000 \mathrm{E}+00 & 0.000 \mathrm{E}+00 & 0.000 \mathrm{E}+00 \\ 23 & 0.000 \mathrm{E}+00 & 0.000 \mathrm{E}+00 & 0.000 \mathrm{E}+00 \\ 24 & 0.000 \mathrm{E}+00 & 0.000 \mathrm{E}+00 & 0.000 \mathrm{E}+00 \\ 25 & 0.000 \mathrm{E}+00 & 0.000 \mathrm{E}+00 & 0.000 \mathrm{E}+00 \\ 26 & 0.000 \mathrm{E}+00 & 0.000 \mathrm{E}+00 & 0.000 \mathrm{E}+00 \\ -\end{array}$

Totals: $2.136 \mathrm{E}+13 \quad 1.638 \mathrm{E}+09 \quad 2.136 \mathrm{E}+13$

\begin{tabular}{|c|c|c|c|c|}
\hline $\begin{array}{l}\text { Nuclide } 492 \text { is } \\
\text { Group Gamma } \\
\text { No. X-ray }\end{array}$ & $\begin{array}{c}(Z=94) \\
\text { Total } \\
\text { photon/sec }\end{array}$ & $\begin{array}{c}\text { Weight (492) } \\
\text { Group Gamma } \\
\text { No. X-ray }\end{array}$ & $\begin{aligned}= & 4.110 \mathrm{E}+0 . \\
& \text { Brems }\end{aligned}$ & $\begin{array}{l}1 \mathrm{Ci} \\
\text { Total } \\
\text { photon/sec }\end{array}$ \\
\hline $\begin{array}{rr}1 & 1.764 \mathrm{E}+11 \\
4 & 6.083 \mathrm{E}+08 \\
10 & 1.521 \mathrm{E}+08\end{array}$ & $\begin{array}{ll}0.000 \mathrm{E}+00 & 1.764 \mathrm{E}+11 \\
0.000 \mathrm{E}+00 & 6.083 \mathrm{E}+08 \\
0.000 \mathrm{E}+00 & 1.521 \mathrm{E}+08\end{array}$ & $\begin{array}{ll}16 & 0.000 \mathrm{E}+00 \\
19 & 0.000 \mathrm{E}+00 \\
25 & 0.000 \mathrm{E}+00\end{array}$ & $\begin{array}{l}0.000 \mathrm{E}+00 \\
0.000 \mathrm{E}+00 \\
0.000 \mathrm{E}+00\end{array}$ & $\begin{array}{l}0.000 E+00 \\
0.000 E+00 \\
0.000 E+00\end{array}$ \\
\hline
\end{tabular}

Totals: $1.772 \mathrm{E}+11 \quad 0.000 \mathrm{E}+00 \quad 1.772 \mathrm{E}+11$

\begin{tabular}{|c|c|c|c|c|c|}
\hline $\begin{array}{l}\text { Juclide } 493 \text { i } \\
\text { aroup Gamma } \\
\text { No. X-ray }\end{array}$ & Brems & $\begin{array}{c}(Z=94) \\
\text { Total } \\
\text { photon/sec }\end{array}$ & $\begin{array}{c}\text { Weight(493) } \\
\text { Group Gamma } \\
\text { No. X-ray }\end{array}$ & Brems & $\begin{array}{l}2 \mathrm{Ci} \\
\text { Total } \\
\text { photon/se }\end{array}$ \\
\hline $\begin{array}{rr}1 & 4.412 \mathrm{E}+11 \\
3 & 2.005 \mathrm{E}+08 \\
4 & 5.715 \mathrm{E}+07 \\
5 & 2.908 \mathrm{E}+07 \\
6 & 3.309 \mathrm{E}+07 \\
7 & 3.108 \mathrm{E}+07 \\
8 & 1.304 \mathrm{E}+06 \\
9 & 2.707 \mathrm{E}+07 \\
10 & 7.520 \mathrm{E}+08 \\
11 & 5.114 \mathrm{E}+07 \\
12 & 3.610 \mathrm{E}+08 \\
13 & 1.705 \mathrm{E}+08 \\
14 & 6.417 \mathrm{E}+06 \\
15 & 1.504 \mathrm{E}+06\end{array}$ & $\begin{array}{l}0.000 \mathrm{E}+00 \\
0.000 \mathrm{E}+00 \\
0.000 \mathrm{E}+00 \\
0.000 \mathrm{E}+00 \\
0.000 \mathrm{E}+00 \\
0.000 \mathrm{E}+00 \\
0.000 \mathrm{E}+00 \\
0.000 \mathrm{E}+00 \\
0.000 \mathrm{E}+00 \\
0.000 \mathrm{E}+00 \\
0.000 \mathrm{E}+00 \\
0.000 \mathrm{E}+00 \\
0.000 \mathrm{E}+00\end{array}$ & $\begin{array}{l}4.412 \mathrm{E}+11 \\
2.005 \mathrm{E}+08 \\
5.715 \mathrm{E}+07 \\
2.908 \mathrm{E}+07 \\
3.309 \mathrm{E}+07 \\
3.108 \mathrm{E}+07 \\
1.304 \mathrm{E}+06 \\
2.707 \mathrm{E}+07 \\
7.520 \mathrm{E}+08 \\
5.114 \mathrm{E}+07 \\
3.610 \mathrm{E}+08 \\
1.705 \mathrm{E}+08 \\
6.417 \mathrm{E}+06\end{array}$ & $\begin{array}{ll}16 & 2.106 \mathrm{E}+04 \\
18 & 0.000 \mathrm{E}+00 \\
19 & 0.000 \mathrm{E}+00 \\
20 & 0.000 \mathrm{E}+00 \\
21 & 0.000 \mathrm{E}+00 \\
22 & 0.000 \mathrm{E}+00 \\
23 & 0.000 \mathrm{E}+00 \\
24 & 0.000 \mathrm{E}+00 \\
25 & 0.000 \mathrm{E}+00 \\
26 & 0.000 \mathrm{E}+00 \\
27 & 0.000 \mathrm{E}+00 \\
28 & 0.000 \mathrm{E}+00 \\
29 & 0.000 \mathrm{E}+00 \\
30 & 0.000 \mathrm{E}+00\end{array}$ & $\begin{array}{l}0.000 E+00 \\
0.000 E+00 \\
0.000 E+00 \\
0.000 E+00 \\
0.000 E+00 \\
0.000 E+00 \\
0.000 E+00 \\
0.000 E+00 \\
0.000 E+00 \\
0.000 E+00 \\
0.000 E+00 \\
0.000 E+00 \\
0.000 E+00\end{array}$ & $\begin{array}{l}2.106 \mathrm{E}+04 \\
0.000 \mathrm{E}+00 \\
0.000 \mathrm{E}+00 \\
0.000 \mathrm{E}+00 \\
0.000 \mathrm{E}+00 \\
0.000 \mathrm{E}+00 \\
0.000 \mathrm{E}+00 \\
0.000 \mathrm{E}+00 \\
0.000 \mathrm{E}+00 \\
0.000 \mathrm{E}+00 \\
0.000 \mathrm{E}+00 \\
0.000 \mathrm{E}+00 \\
0.000 \mathrm{E}+00 \\
0.000 \mathrm{E}+00\end{array}$ \\
\hline
\end{tabular}


HNF-SD-WM-CN-104

Rev. 0

Page 13 of 33

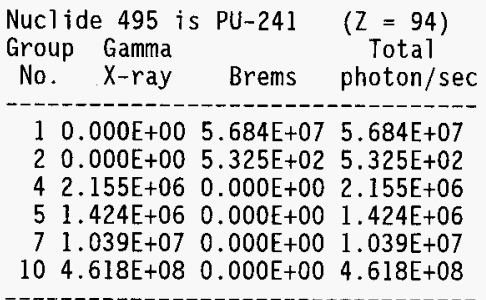

\begin{tabular}{cccc}
$\begin{array}{c}\text { Weight }(495)=1.040 \mathrm{E}+03 \mathrm{Ci} \\
\text { Group Gamma } \\
\text { Total } \\
\text { No. X-ray }\end{array}$ & $\begin{array}{c}\text { Brems } \\
\text { photon } / \mathrm{sec}\end{array}$ \\
\hdashline 16 & $0.000 \mathrm{E}+00$ & $0.000 \mathrm{E}+00$ & $0.000 \mathrm{E}+00$ \\
17 & $0.000 \mathrm{E}+00$ & $0.000 \mathrm{E}+00$ & $0.000 \mathrm{E}+00$ \\
19 & $0.000 \mathrm{E}+00$ & $0.000 \mathrm{E}+00$ & $0.000 \mathrm{E}+00$ \\
20 & $0.000 \mathrm{E}+00$ & $0.000 \mathrm{E}+00$ & $0.000 \mathrm{E}+00$ \\
22 & $0.000 \mathrm{E}+00$ & $0.000 \mathrm{E}+00$ & $0.000 \mathrm{E}+00$ \\
25 & $0.000 \mathrm{E}+00$ & $0.000 \mathrm{E}+00$ & $0.000 \mathrm{E}+00$
\end{tabular}

Totals: $4.757 \mathrm{E}+08 \quad 5.684 \mathrm{E}+07 \quad 5.326 \mathrm{E}+08$

\begin{tabular}{rccc}
$\begin{array}{c}\text { Nuclide } \\
\text { Group Gamma }\end{array}$ & $\begin{array}{c}\text { G } \\
\text { No. }\end{array}$ X-ray & Brems & $\begin{array}{c}(Z=95) \\
\text { Total } \\
\text { photon/sec }\end{array}$ \\
\hdashline 1 & $1.074 \mathrm{E}+14$ & $4.177 \mathrm{E}+09$ & $1.074 \mathrm{E}+14$ \\
2 & $5.994 \mathrm{E}+12$ & $4.337 \mathrm{E}+08$ & $5.994 \mathrm{E}+12$ \\
3 & $2.747 \mathrm{E}+11$ & $5.112 \mathrm{E}+07$ & $2.748 \mathrm{E}+11$ \\
4 & $2.248 \mathrm{E}+11$ & $5.589 \mathrm{E}+06$ & $2.248 \mathrm{E}+11$ \\
5 & $8.916 \mathrm{E}+13$ & $8.508 \mathrm{E}+05$ & $8.916 \mathrm{E}+13$ \\
6 & $4.995 \mathrm{E}+09$ & $1.611 \mathrm{E}+05$ & $4.995 \mathrm{E}+09$ \\
7 & $0.000 \mathrm{E}+00$ & $2.304 \mathrm{E}+04$ & $2.304 \mathrm{E}+04$ \\
8 & $0.000 \mathrm{E}+00$ & $1.532 \mathrm{E}+03$ & $1.532 \mathrm{E}+03$ \\
9 & $5.245 \mathrm{E}+10$ & $1.085 \mathrm{E}+01$ & $5.245 \mathrm{E}+10$ \\
10 & $6.993 \mathrm{E}+10$ & $0.000 \mathrm{E}+00$ & $6.993 \mathrm{E}+10$ \\
11 & $2.148 \mathrm{E}+09$ & $0.000 \mathrm{E}+00$ & $2.148 \mathrm{E}+09$ \\
12 & $3.247 \mathrm{E}+09$ & $0.000 \mathrm{E}+00$ & $3.247 \mathrm{E}+09$ \\
13 & $1.948 \mathrm{E}+08$ & $0.000 \mathrm{E}+00$ & $1.948 \mathrm{E}+08$ \\
14 & $1.723 \mathrm{E}+09$ & $0.000 \mathrm{E}+00$ & $1.723 \mathrm{E}+09$ \\
15 & $5.494 \mathrm{E}+07$ & $0.000 \mathrm{E}+00$ & $5.494 \mathrm{E}+07$
\end{tabular}

Weight (496) $=6.750 \mathrm{E}+03 \mathrm{Ci}$ Group Gamma Total No. X-ray Brems photon/sec

$160.000 E+00 \quad 0.000 E+00 \quad 0.000 E+00$

$17 \quad 0.000 \mathrm{E}+00 \quad 0.000 \mathrm{E}+00 \quad 0.000 \mathrm{E}+00$

$18 \quad 0.000 \mathrm{E}+00 \quad 0.000 \mathrm{E}+00 \quad 0.000 \mathrm{E}+00$

$190.000 \mathrm{E}+00 \quad 0.000 \mathrm{E}+00 \quad 0.000 \mathrm{E}+00$

$20 \quad 0.000 \mathrm{E}+00 \quad 0.000 \mathrm{E}+00 \quad 0.000 \mathrm{E}+00$

2] $0.000 \mathrm{E}+00 \quad 0.000 \mathrm{E}+00 \quad 0.000 \mathrm{E}+00$

$220.000 \mathrm{E}+00 \quad 0.000 \mathrm{E}+00 \quad 0.000 \mathrm{E}+00$

$230.000 \mathrm{E}+00 \quad 0.000 \mathrm{E}+00 \quad 0.000 \mathrm{E}+00$

$240.000 \mathrm{E}+00 \quad 0.000 \mathrm{E}+00 \quad 0.000 \mathrm{E}+00$

$250.000 \mathrm{E}+00 \quad 0.000 \mathrm{E}+00 \quad 0.000 \mathrm{E}+00$

$260.000 \mathrm{E}+00 \quad 0.000 \mathrm{E}+00 \quad 0.000 \mathrm{E}+00$

$270.000 \mathrm{E}+00 \quad 0.000 \mathrm{E}+00 \quad 0.000 \mathrm{E}+00$

$280.000 \mathrm{E}+00 \quad 0.000 \mathrm{E}+00 \quad 0.000 \mathrm{E}+00$

$290.000 \mathrm{E}+00 \quad 0.000 \mathrm{E}+00 \quad 0.000 \mathrm{E}+00$

$30 \quad 0.000 \mathrm{E}+00 \quad 0.000 \mathrm{E}+00 \quad 0.000 \mathrm{E}+00$

Totals: $2.032 \mathrm{E}+14 \quad 4.668 \mathrm{E}+092.032 \mathrm{E}+14$

\begin{tabular}{|c|c|c|c|c|c|c|}
\hline $\begin{array}{l}\text { Nuclide } \\
\text { Group } \\
\text { No. }\end{array}$ & $\begin{array}{l}500 \text { is } \\
\text { Gamma } \\
\text { X-ray }\end{array}$ & CM-244 & $\begin{array}{c}(Z=96) \\
\text { Total } \\
\text { photon } / \mathrm{sec}\end{array}$ & $\begin{array}{c}\text { Weight }(500) \\
\text { Group Gamma } \\
\text { No. X-ray }\end{array}$ & $=3.740 E+01$ & $\begin{array}{l}1 \mathrm{Ci} \\
\text { Total } \\
\text { photon/sec }\end{array}$ \\
\hline $\begin{array}{ll}1 & 2 . \\
4 & 1 .\end{array}$ & $\begin{array}{l}491 \mathrm{E}+11 \\
245 \mathrm{E}+09\end{array}$ & $\begin{array}{l}0.000 E+00 \\
0.000 E+00\end{array}$ & $\begin{array}{l}2.491 E+11 \\
1.245 E+09\end{array}$ & $\begin{array}{ll}16 & 0.000 E+00 \\
19 & 0.000 E+00\end{array}$ & $\begin{array}{l}0.000 E+00 \\
0.000 E+00\end{array}$ & $\begin{array}{l}0.000 E+00 \\
0.000 E+00\end{array}$ \\
\hline
\end{tabular}

Totals: $2.503 \mathrm{E}+11 \quad 0.000 \mathrm{E}+00 \quad 2.503 \mathrm{E}+11$ 


\section{HNF-SD-WM-CN-104 \\ Rev. 0 \\ Page 14 of 33}

UNMITIGATED 12 HOUR RELEASE - 100 M AWAY

$\begin{array}{ccl}\text { Source } & \text { Shields } & \text { Distance to Detector } X=1.023 \mathrm{E}+04 \mathrm{~cm} \\ \text { Slab } & \text { Slab } & \text { Source Volume }=1.703 \mathrm{E}+08 \mathrm{cc} \\ & & \text { Source Mass }=3.677 \mathrm{E}+08 \text { grams }\end{array}$

Thickness $=2.134 \mathrm{E}+02 \mathrm{~cm} \quad$ Height $=6.096 \mathrm{E}+01 \mathrm{~cm} \quad$ Width $=1.309 \mathrm{E}+04 \mathrm{~cm}$

Integration Specs: $\quad$ NTHETA $=15 \quad$ NPSI $=15 \quad$ DELR $=5.080 \mathrm{E}+00 \mathrm{~cm}$

Total Intervals: $9.450 \mathrm{E}+03$ (photon source is the lst region)

Shield Thickness: $2.134 E+02,1.524 E+01,1.000 E+04 \mathrm{~cm}$

Distances from Dose Point to the Outside of

(1) Source Region: $1.002 E+04 \mathrm{~cm}$ (2) Next Layer: $1.000 \mathrm{E}+04 \mathrm{~cm}$

Dose Buildup Data for Shield 2 with Effective Atomic Number 10.78

Fluence-to-Dose Conversion: Photons in Air

\begin{tabular}{|c|c|c|c|c|}
\hline $\begin{array}{l}\text { Average } \\
\mathrm{E}, \mathrm{MeV}\end{array}$ & $\begin{array}{r}\text { Source Total } \\
\text { photons/sec }\end{array}$ & $\begin{array}{l}\text { Fluence to } \\
\text { Dose Factor }\end{array}$ & $\begin{array}{l}\text { Energy Fluence } \\
\mathrm{MeV} / \mathrm{sq} . \mathrm{cm} / \mathrm{sec}\end{array}$ & $\begin{array}{c}\text { Dose Rate } \\
\mathrm{R} / \mathrm{hr}\end{array}$ \\
\hline $\begin{array}{l}0.015 \\
0.025 \\
0.035 \\
0.045 \\
0.055 \\
0.065 \\
0.075 \\
0.085 \\
0.095 \\
0.150 \\
0.250 \\
0.350 \\
0.475 \\
0.650 \\
0.825 \\
1.000 \\
1.225 \\
1.475 \\
1.700 \\
1.900 \\
2.100 \\
2.300\end{array}$ & $\begin{array}{l}2.286 \mathrm{E}+15 \\
1.138 \mathrm{E}+15 \\
1.123 \mathrm{E}+15 \\
5.394 \mathrm{E}+14 \\
4.339 \mathrm{E}+14 \\
2.938 \mathrm{E}+14 \\
2.667 \mathrm{E}+14 \\
2.451 \mathrm{E}+14 \\
2.193 \mathrm{E}+14 \\
1.195 \mathrm{E}+15 \\
3.547 \mathrm{E}+14 \\
1.640 \mathrm{E}+14 \\
1.273 \mathrm{E}+14 \\
5.487 \mathrm{E}+15 \\
6.249 \mathrm{E}+13 \\
8.945 \mathrm{E}+13 \\
1.247 \mathrm{E}+14 \\
1.201 \mathrm{E}+13 \\
2.980 \mathrm{E}+12 \\
1.610 \mathrm{E}+11 \\
1.062 \mathrm{E}+11 \\
1.981 \mathrm{E}+08\end{array}$ & $\begin{array}{l}8.183 \mathrm{E}-05 \\
1.656 \mathrm{E}-05 \\
6.084 \mathrm{E}-06 \\
3.203 \mathrm{E}-06 \\
2.216 \mathrm{E}-06 \\
1.827 \mathrm{E}-06 \\
1.664 \mathrm{E}-06 \\
1.603 \mathrm{E}-06 \\
1.592 \mathrm{E}-06 \\
1.728 \mathrm{E}-06 \\
1.935 \mathrm{E}-06 \\
2.019 \mathrm{E}-06 \\
2.043 \mathrm{E}-06 \\
2.029 \mathrm{E}-06 \\
1.983 \mathrm{E}-06 \\
1.923 \mathrm{E}-06 \\
1.850 \mathrm{E}-06 \\
1.775 \mathrm{E}-06 \\
1.712 \mathrm{E}-06 \\
1.660 \mathrm{E}-06 \\
1.613 \mathrm{E}-06 \\
1.592 \mathrm{E}-06\end{array}$ & $\begin{array}{l}0.000 \mathrm{E}+00 \\
2.473 \mathrm{E}-28 \\
3.183 \mathrm{E}-09 \\
8.309 \mathrm{E}-04 \\
1.144 \mathrm{E}-01 \\
9.278 \mathrm{E}-01 \\
3.991 \mathrm{E}+00 \\
9.083 \mathrm{E}+00 \\
1.607 \mathrm{E}+01 \\
2.456 \mathrm{E}+02 \\
2.831 \mathrm{E}+02 \\
2.666 \mathrm{E}+02 \\
3.629 \mathrm{E}+02 \\
2.757 \mathrm{E}+04 \\
4.929 \mathrm{E}+02 \\
1.013 \mathrm{E}+03 \\
2.030 \mathrm{E}+03 \\
2.713 \mathrm{E}+02 \\
8.682 \mathrm{E}+01 \\
5.729 \mathrm{E}+00 \\
4.511 \mathrm{E}+00 \\
9.855 \mathrm{E}-03\end{array}$ & $\begin{array}{l}0.000 \mathrm{E}+00 \\
4.096 \mathrm{E}-33 \\
1.937 \mathrm{E}-14 \\
2.661 \mathrm{E}-09 \\
2.535 \mathrm{E}-07 \\
1.695 \mathrm{E}-06 \\
6.642 \mathrm{E}-06 \\
1.456 \mathrm{E}-05 \\
2.558 \mathrm{E}-05 \\
4.244 \mathrm{E}-04 \\
5.477 \mathrm{E}-04 \\
5.383 \mathrm{E}-04 \\
7.413 \mathrm{E}-04 \\
5.594 \mathrm{E}-02 \\
9.775 \mathrm{E}-04 \\
1.948 \mathrm{E}-03 \\
3.755 \mathrm{E}-03 \\
4.816 \mathrm{E}-04 \\
1.486 \mathrm{E}-04 \\
9.509 \mathrm{E}-06 \\
7.276 \mathrm{E}-06 \\
1.569 \mathrm{E}-08\end{array}$ \\
\hline Totals: & $1.416 \mathrm{E}+16 \mathrm{p}$ & ons/sec & $3.266 \mathrm{E}+04$ & $\begin{array}{l}6.557 \mathrm{E}-02 \\
4.699 \mathrm{E}-09\end{array}$ \\
\hline
\end{tabular}


HNF-SD-WM-CN-104

Rev. 0

Page 15 of 33

Dose Equivalent Rates (rem/hr) for Other Fluence-to-Dose Factors

Average Anterior Posterior Lateral Rotational Isometric Orig ANSI $\mathrm{E}, \mathrm{MeV}$ DUNIT $=1$ DUNIT $=2 \quad$ DUNIT $=3 \quad$ DUNIT $=4 \quad$ DUNIT $=5 \quad$ DUNIT $=6$

$\begin{array}{llllllr}0.025 & 1.026 \mathrm{E}-33 & 4.690 \mathrm{E}-34 & 2.431 \mathrm{E}-34 & 4.990 \mathrm{E}-34 & 4.112 \mathrm{E}-34 & 7.916 \mathrm{E}-33 \\ 0.035 & 1.120 \mathrm{E}-14 & 6.484 \mathrm{E}-15 & 3.426 \mathrm{E}-15 & 5.950 \mathrm{E}-15 & 4.834 \mathrm{E}-15 & 3.981 \mathrm{E}-14 \\ 0.045 & 2.475 \mathrm{E}-09 & 1.582 \mathrm{E}-09 & 9.039 \mathrm{E}-10 & 1.401 \mathrm{E}-09 & 1.133 \mathrm{E}-09 & 5.855 \mathrm{E}-09 \\ 0.055 & 2.973 \mathrm{E}-07 & 2.014 \mathrm{E}-07 & 1.214 \mathrm{E}-07 & 1.760 \mathrm{E}-07 & 1.425 \mathrm{E}-07 & 5.703 \mathrm{E}-07 \\ 0.065 & 2.167 \mathrm{E}-06 & 1.533 \mathrm{E}-06 & 9.475 \mathrm{E}-07 & 1.326 \mathrm{E}-06 & 1.078 \mathrm{E}-06 & 3.704 \mathrm{E}-06 \\ 0.075 & 8.600 \mathrm{E}-06 & 6.304 \mathrm{E}-06 & 3.919 \mathrm{E}-06 & 5.397 \mathrm{E}-06 & 4.408 \mathrm{E}-06 & 1.375 \mathrm{E}-05 \\ 0.085 & 1.842 \mathrm{E}-05 & 1.393 \mathrm{E}-05 & 8.619 \mathrm{E}-06 & 1.181 \mathrm{E}-05 & 9.677 \mathrm{E}-06 & 2.828 \mathrm{E}-05 \\ 0.095 & 3.117 \mathrm{E}-05 & 2.422 \mathrm{E}-05 & 1.485 \mathrm{E}-05 & 2.035 \mathrm{E}-05 & 1.671 \mathrm{E}-05 & 4.664 \mathrm{E}-05 \\ 0.150 & 4.423 \mathrm{E}-04 & 3.693 \mathrm{E}-04 & 2.340 \mathrm{E}-04 & 3.168 \mathrm{E}-04 & 2.577 \mathrm{E}-04 & 6.211 \mathrm{E}-04 \\ 0.250 & 5.295 \mathrm{E}-04 & 4.379 \mathrm{E}-04 & 2.945 \mathrm{E}-04 & 3.813 \mathrm{E}-04 & 3.149 \mathrm{E}-04 & 7.144 \mathrm{E}-04 \\ 0.350 & 4.986 \mathrm{E}-04 & 4.191 \mathrm{E}-04 & 2.919 \mathrm{E}-04 & 3.685 \mathrm{E}-04 & 3.076 \mathrm{E}-04 & 6.688 \mathrm{E}-04 \\ 0.475 & 6.669 \mathrm{E}-04 & 5.731 \mathrm{E}-04 & 4.118 \mathrm{E}-04 & 5.080 \mathrm{E}-04 & 4.285 \mathrm{E}-04 & 8.531 \mathrm{E}-04 \\ 0.650 & 4.894 \mathrm{E}-02 & 4.312 \mathrm{E}-02 & 3.199 \mathrm{E}-02 & 3.851 \mathrm{E}-02 & 3.287 \mathrm{E}-02 & 6.117 \mathrm{E}-02 \\ 0.825 & 8.426 \mathrm{E}-04 & 7.565 \mathrm{E}-04 & 5.748 \mathrm{E}-04 & 6.789 \mathrm{E}-04 & 5.852 \mathrm{E}-04 & 1.028 \mathrm{E}-03 \\ 1.000 & 1.668 \mathrm{E}-03 & 1.519 \mathrm{E}-03 & 1.177 \mathrm{E}-03 & 1.368 \mathrm{E}-03 & 1.189 \mathrm{E}-03 & 2.004 \mathrm{E}-03 \\ 1.225 & 3.197 \mathrm{E}-03 & 2.949 \mathrm{E}-03 & 2.333 \mathrm{E}-03 & 2.663 \mathrm{E}-03 & 2.338 \mathrm{E}-03 & 3.789 \mathrm{E}-03 \\ 1.475 & 4.083 \mathrm{E}-04 & 3.805 \mathrm{E}-04 & 3.067 \mathrm{E}-04 & 3.444 \mathrm{E}-04 & 3.053 \mathrm{E}-04 & 4.792 \mathrm{E}-04 \\ 1.700 & 1.258 \mathrm{E}-04 & 1.180 \mathrm{E}-04 & 9.651 \mathrm{E}-05 & 1.070 \mathrm{E}-04 & 9.555 \mathrm{E}-05 & 1.467 \mathrm{E}-04 \\ 1.900 & 8.051 \mathrm{E}-06 & 7.585 \mathrm{E}-06 & 6.271 \mathrm{E}-06 & 6.887 \mathrm{E}-06 & 6.183 \mathrm{E}-06 & 9.342 \mathrm{E}-06 \\ 2.100 & 6.161 \mathrm{E}-06 & 5.825 \mathrm{E}-06 & 4.863 \mathrm{E}-06 & 5.295 \mathrm{E}-06 & 4.778 \mathrm{E}-06 & 7.120 \mathrm{E}-06 \\ 2.300 & 1.311 \mathrm{E}-08 & 1.243 \mathrm{E}-08 & 1.047 \mathrm{E}-08 & 1.131 \mathrm{E}-08 & 1.025 \mathrm{E}-08 & 1.509 \mathrm{E}-08 \\ -1.08 & & & & & & \end{array}$

Fluence-to-Dose Factors, rem/hr per Mev/sq. cm/sec

Average Anterior Posterior Lateral Rotational Isometric Orig ANSI $\mathrm{E}$, Mev DUNIT $=1 \quad$ DUNIT $=2 \quad$ DUNIT $=3 \quad$ DUNIT $=4 \quad$ DUNIT $=5 \quad$ DUNIT $=6$

$\begin{array}{lllllll}0.025 & 4.148 \mathrm{E}-06 & 1.896 \mathrm{E}-06 & 9.827 \mathrm{E}-07 & 2.017 \mathrm{E}-06 & 1.663 \mathrm{E}-06 & 3.201 \mathrm{E}-05 \\ 0.035 & 3.518 \mathrm{E}-06 & 2.037 \mathrm{E}-06 & 1.076 \mathrm{E}-06 & 1.869 \mathrm{E}-06 & 1.519 \mathrm{E}-06 & 1.251 \mathrm{E}-05 \\ 0.045 & 2.979 \mathrm{E}-06 & 1.904 \mathrm{E}-06 & 1.088 \mathrm{E}-06 & 1.686 \mathrm{E}-06 & 1.363 \mathrm{E}-06 & 7.047 \mathrm{E}-06 \\ 0.055 & 2.598 \mathrm{E}-06 & 1.760 \mathrm{E}-06 & 1.061 \mathrm{E}-06 & 1.538 \mathrm{E}-06 & 1.245 \mathrm{E}-06 & 4.985 \mathrm{E}-06 \\ 0.065 & 2.336 \mathrm{E}-06 & 1.652 \mathrm{E}-06 & 1.021 \mathrm{E}-06 & 1.429 \mathrm{E}-06 & 1.162 \mathrm{E}-06 & 3.993 \mathrm{E}-06 \\ 0.075 & 2.155 \mathrm{E}-06 & 1.580 \mathrm{E}-06 & 9.820 \mathrm{E}-07 & 1.352 \mathrm{E}-06 & 1.104 \mathrm{E}-06 & 3.444 \mathrm{E}-06 \\ 0.085 & 2.028 \mathrm{E}-06 & 1.534 \mathrm{E}-06 & 9.489 \mathrm{E}-07 & 1.300 \mathrm{E}-06 & 1.065 \mathrm{E}-06 & 3.113 \mathrm{E}-06 \\ 0.095 & 1.940 \mathrm{E}-06 & 1.507 \mathrm{E}-06 & 9.243 \mathrm{E}-07 & 1.266 \mathrm{E}-06 & 1.040 \mathrm{E}-06 & 2.903 \mathrm{E}-06 \\ 0.150 & 1.801 \mathrm{E}-06 & 1.504 \mathrm{E}-06 & 9.526 \mathrm{E}-07 & 1.290 \mathrm{E}-06 & 1.049 \mathrm{E}-06 & 2.529 \mathrm{E}-06 \\ 0.250 & 1.870 \mathrm{E}-06 & 1.547 \mathrm{E}-06 & 1.040 \mathrm{E}-06 & 1.347 \mathrm{E}-06 & 1.113 \mathrm{E}-06 & 2.524 \mathrm{E}-06 \\ 0.350 & 1.870 \mathrm{E}-06 & 1.572 \mathrm{E}-06 & 1.095 \mathrm{E}-06 & 1.382 \mathrm{E}-06 & 1.154 \mathrm{E}-06 & 2.509 \mathrm{E}-06 \\ 0.475 & 1.838 \mathrm{E}-06 & 1.579 \mathrm{E}-06 & 1.135 \mathrm{E}-06 & 1.400 \mathrm{E}-06 & 1.181 \mathrm{E}-06 & 2.351 \mathrm{E}-06 \\ 0.650 & 1.775 \mathrm{E}-06 & 1.564 \mathrm{E}-06 & 1.160 \mathrm{E}-06 & 1.397 \mathrm{E}-06 & 1.192 \mathrm{E}-06 & 2.219 \mathrm{E}-06 \\ 0.825 & 1.709 \mathrm{E}-06 & 1.535 \mathrm{E}-06 & 1.166 \mathrm{E}-06 & 1.377 \mathrm{E}-06 & 1.187 \mathrm{E}-06 & 2.086 \mathrm{E}-06 \\ 1.000 & 1.647 \mathrm{E}-06 & 1.500 \mathrm{E}-06 & 1.162 \mathrm{E}-06 & 1.350 \mathrm{E}-06 & 1.174 \mathrm{E}-06 & 1.979 \mathrm{E}-06 \\ 1.225 & 1.575 \mathrm{E}-06 & 1.453 \mathrm{E}-06 & 1.149 \mathrm{E}-06 & 1.312 \mathrm{E}-06 & 1.152 \mathrm{E}-06 & 1.867 \mathrm{E}-06 \\ 1.475 & 1.505 \mathrm{E}-06 & 1.402 \mathrm{E}-06 & 1.130 \mathrm{E}-06 & 1.269 \mathrm{E}-06 & 1.125 \mathrm{E}-06 & 1.766 \mathrm{E}-06 \\ 1.700 & 1.449 \mathrm{E}-06 & 1.359 \mathrm{E}-06 & 1.112 \mathrm{E}-06 & 1.233 \mathrm{E}-06 & 1.100 \mathrm{E}-06 & 1.690 \mathrm{E}-06\end{array}$


HNF-SD-WM-CN-104

Rev. 0

Page 16 of 33

$\begin{array}{lllllll}1.900 & 1.405 \mathrm{E}-06 & 1.324 \mathrm{E}-06 & 1.095 \mathrm{E}-06 & 1.202 \mathrm{E}-06 & 1.079 \mathrm{E}-06 & 1.631 \mathrm{E}-06 \\ 2.100 & 1.366 \mathrm{E}-06 & 1.291 \mathrm{E}-06 & 1.078 \mathrm{E}-06 & 1.174 \mathrm{E}-06 & 1.059 \mathrm{E}-06 & 1.578 \mathrm{E}-06 \\ 2.300 & 1.330 \mathrm{E}-06 & 1.261 \mathrm{E}-06 & 1.062 \mathrm{E}-06 & 1.147 \mathrm{E}-06 & 1.040 \mathrm{E}-06 & 1.531 \mathrm{E}-06\end{array}$

MITIGATEd 12 HOUR RELEASE - 100 M AWAY

Table of Source Activity:

Scale Factor $=1.000 \mathrm{E}+00$

\begin{tabular}{|c|c|c|}
\hline $\begin{array}{l}\text { I sotope } \\
\text { Name }\end{array}$ & $\begin{array}{l}\text { Initial } \\
\text { Values }\end{array}$ & $\begin{array}{l}\text { Final } \\
\text { Curies }\end{array}$ \\
\hline $\begin{array}{l}C O-60 \\
S R-90 \\
Y-90 \\
C S-137 \\
\text { BA-137M } \\
\text { EU-154 } \\
\text { NP-237 } \\
\text { PU-238 } \\
\text { PU-239 } \\
P U-241 \\
\text { AM-241 } \\
\text { CM-244 }\end{array}$ & $\begin{array}{l}2.64 \mathrm{E}+02 \\
1.07 \mathrm{E}+06 \\
1.07 \mathrm{E}+06 \\
1.62 \mathrm{E}+05 \\
1.53 \mathrm{E}+05 \\
4.17 \mathrm{E}+03 \\
7.74 \mathrm{E}+01 \\
1.09 \mathrm{E}+02 \\
2.76 \mathrm{E}+02 \\
1.90 \mathrm{E}+03 \\
8.08 \mathrm{E}+02 \\
5.08 \mathrm{E}+00\end{array}$ & $\begin{array}{l}2.640 \mathrm{E}+02 \\
1.070 \mathrm{E}+06 \\
1.070 \mathrm{E}+06 \\
1.620 \mathrm{E}+05 \\
1.530 \mathrm{E}+05 \\
4.170 \mathrm{E}+03 \\
7.740 \mathrm{E}+01 \\
1.090 \mathrm{E}+02 \\
2.760 \mathrm{E}+02 \\
1.900 \mathrm{E}+03 \\
8.080 \mathrm{E}+02 \\
5.080 \mathrm{E}+00\end{array}$ \\
\hline
\end{tabular}


MITIGATED 12 HOUR RELEASE - 100 M AWAY

Shield Composition, g/cc

Shield 1 Shield 2 Shield 3

\begin{tabular}{llll} 
WATER & $5.600 \mathrm{E}-01$ & $0.000 \mathrm{E}+00$ & $0.000 \mathrm{E}+00$ \\
AIR & $0.000 \mathrm{E}+00$ & $0.000 \mathrm{E}+00$ & $1.290 \mathrm{E}-03$ \\
HAN SOIL & $1.600 \mathrm{E}+00$ & $1.600 \mathrm{E}+00$ & $0.000 \mathrm{E}+00$ \\
\hline Totals: & $2.160 \mathrm{E}+00$ & $1.600 \mathrm{E}+00$ & $1.290 \mathrm{E}-03$
\end{tabular}

$\mathrm{E}$, MeV Linear Attenuation, per cm (last region is air)

$\begin{array}{cccc}0.015 & 2.044 \mathrm{E}+01 & 1.952 \mathrm{E}+01 & 2.046 \mathrm{E}-03 \\ 0.025 & 4.813 \mathrm{E}+00 & 4.535 \mathrm{E}+00 & 6.138 \mathrm{E}-04 \\ 0.035 & 1.986 \mathrm{E}+00 & 1.817 \mathrm{E}+00 & 3.664 \mathrm{E}-04 \\ 0.045 & 1.119 \mathrm{E}+00 & 9.853 \mathrm{E}-01 & 2.837 \mathrm{E}-04 \\ 0.055 & 7.679 \mathrm{E}-01 & 6.493 \mathrm{E}-01 & 2.491 \mathrm{E}-04 \\ 0.065 & 5.980 \mathrm{E}-01 & 4.872 \mathrm{E}-01 & 2.314 \mathrm{E}-04 \\ 0.075 & 5.029 \mathrm{E}-01 & 3.981 \mathrm{E}-01 & 2.184 \mathrm{E}-04 \\ 0.085 & 4.446 \mathrm{E}-01 & 3.442 \mathrm{E}-01 & 2.090 \mathrm{E}-04 \\ 0.095 & 4.062 \mathrm{E}-01 & 3.091 \mathrm{E}-01 & 2.019 \mathrm{E}-04 \\ 0.150 & 3.135 \mathrm{E}-01 & 2.288 \mathrm{E}-01 & 1.758 \mathrm{E}-04 \\ 0.250 & 2.546 \mathrm{E}-01 & 1.835 \mathrm{E}-01 & 1.477 \mathrm{E}-04 \\ 0.350 & 2.229 \mathrm{E}-01 & 1.603 \mathrm{E}-01 & 1.298 \mathrm{E}-04 \\ 0.475 & 1.961 \mathrm{E}-01 & 1.408 \mathrm{E}-01 & 1.146 \mathrm{E}-04 \\ 0.650 & 1.710 \mathrm{E}-01 & 1.227 \mathrm{E}-01 & 1.001 \mathrm{E}-04 \\ 0.825 & 1.533 \mathrm{E}-01 & 1.099 \mathrm{E}-01 & 8.984 \mathrm{E}-05 \\ 1.000 & 1.397 \mathrm{E}-01 & 1.002 \mathrm{E}-01 & 8.194 \mathrm{E}-05 \\ 1.225 & 1.263 \mathrm{E}-01 & 9.058 \mathrm{E}-02 & 7.398 \mathrm{E}-05 \\ 1.475 & 1.150 \mathrm{E}-01 & 8.246 \mathrm{E}-02 & 6.718 \mathrm{E}-05 \\ 1.700 & 1.068 \mathrm{E}-01 & 7.669 \mathrm{E}-02 & 6.249 \mathrm{E}-05 \\ 1.900 & 1.008 \mathrm{E}-01 & 7.243 \mathrm{E}-02 & 5.889 \mathrm{E}-05 \\ 2.100 & 9.574 \mathrm{E}-02 & 6.882 \mathrm{E}-02 & 5.564 \mathrm{E}-05 \\ 2.300 & 9.133 \mathrm{E}-02 & 6.573 \mathrm{E}-02 & 5.263 \mathrm{E}-05 \\ 2.500 & 8.750 \mathrm{E}-02 & 6.304 \mathrm{E}-02 & 5.003 \mathrm{E}-05 \\ 2.700 & 8.418 \mathrm{E}-02 & 6.072 \mathrm{E}-02 & 4.778 \mathrm{E}-05 \\ 3.000 & 7.995 \mathrm{E}-02 & 5.776 \mathrm{E}-02 & 4.511 \mathrm{E}-05 \\ 3.600 & 7.338 \mathrm{E}-02 & 5.323 \mathrm{E}-02 & 4.158 \mathrm{E}-05 \\ 4.500 & 6.631 \mathrm{E}-02 & 4.842 \mathrm{E}-02 & 3.746 \mathrm{E}-05 \\ 5.500 & 6.089 \mathrm{E}-02 & 4.474 \mathrm{E}-02 & 3.381 \mathrm{E}-05 \\ 6.600 & 5.677 \mathrm{E}-02 & 4.198 \mathrm{E}-02 & 3.111 \mathrm{E}-05 \\ 8.600 & 5.210 \mathrm{E}-02 & 3.898 \mathrm{E}-02 & 2.771 \mathrm{E}-05 \\ ----0-------------------- & \end{array}$


HNF-SD-WM-CN-104

Rev. 0

Page 18 of 33

\section{MITIGATED 12 HOUR RELEASE - $100 \mathrm{M}$ AWAY}

Photon Production Rate for Each Radionuclide:

\begin{tabular}{|c|c|c|c|c|c|c|c|}
\hline $\begin{array}{l}\text { Nuclid } \\
\text { Group } \\
\text { No. }\end{array}$ & $\begin{array}{c}\text { de } 472 \text { is } \\
\text { Gamma } \\
\text { X-ray }\end{array}$ & Brems & $\begin{array}{c}(Z=27) \\
\text { Total } \\
\text { photon/sec }\end{array}$ & $\begin{array}{l}\text { Weigl } \\
\text { Group } \\
\text { No. }\end{array}$ & $\begin{array}{r}\text { ht (472) }= \\
\text { Gamma } \\
\text { X-ray }\end{array}$ & $=2.640 \mathrm{E}+02$ & $\begin{array}{l}2 \mathrm{Ci} \\
\text { Total } \\
\text { photon/sec }\end{array}$ \\
\hline $\begin{array}{rl}1 & 0 \\
2 & 0 \\
3 & 0 \\
4 & 0 \\
5 & 0 \\
6 & 0 \\
7 & 0 \\
8 & 0 \\
9 & 0 \\
10 & 0 \\
11 & 0 \\
12 & 0 \\
15 & 9\end{array}$ & & $\begin{array}{l}8 . \\
4 . \\
1 . \\
1 . \\
8 . \\
6 . \\
4 . \\
3 .\end{array}$ & & $\begin{array}{ll}16 & 0 \\
17 & 1 \\
18 & 0 \\
19 & 0 \\
20 & 0 \\
21 & 0 \\
22 & 0 \\
23 & 0 \\
24 & 0 \\
25 & 0 \\
26 & 0 \\
27 & 0 \\
30 & 0\end{array}$ & & & $\begin{array}{l}0.000 \mathrm{E}+00 \\
1.954 \mathrm{E}+13 \\
0.000 \mathrm{E}+00 \\
0.000 \mathrm{E}+00 \\
0.000 \mathrm{E}+00 \\
0.000 \mathrm{E}+00 \\
0.000 \mathrm{E}+00 \\
0.000 \mathrm{E}+00 \\
0.000 \mathrm{E}+00 \\
0.000 \mathrm{E}+00 \\
0.000 \mathrm{E}+00 \\
0.000 \mathrm{E}+00 \\
0.000 \mathrm{E}+00\end{array}$ \\
\hline
\end{tabular}

Tota7s: $1.954 \mathrm{E}+13 \quad 4.740 \mathrm{E}+10 \quad 1.958 \mathrm{E}+13$

\begin{tabular}{|c|c|c|c|c|c|c|c|}
\hline $\begin{array}{l}\text { Nucl id } \\
\text { Group } \\
\text { No. }\end{array}$ & $\begin{array}{l}\text { de } 82 \text { is } \\
\text { Gamma } \\
\text { X-ray }\end{array}$ & & $\begin{array}{c}(Z=38) \\
\text { Total } \\
\text { photon/sec }\end{array}$ & $\begin{array}{l}\text { Weigh } \\
\text { Group } \\
\text { No. }\end{array}$ & $\begin{array}{l}\text { ht ( 82) } \\
\text { Gamma } \\
\text { X-ray }\end{array}$ & Brems & $\begin{array}{l}6 \mathrm{Ci} \\
\text { Total } \\
\text { photon/sec }\end{array}$ \\
\hline $\begin{array}{rl}1 & 0 . \\
2 & 0 . \\
3 & 0 . \\
4 & 0 . \\
5 & 0 . \\
6 & 0 . \\
7 & 0 . \\
8 & 0 . \\
9 & 0 . \\
10 & 0 . \\
11 & 0 . \\
12 & 0 . \\
13 & 0 .\end{array}$ & & $\begin{array}{l}1.67 \\
1.03 \\
5.84 \\
2.93\end{array}$ & & $\begin{array}{ll}16 & 0 \\
17 & 0 \\
18 & 0 \\
19 & 0 \\
20 & 0 \\
21 & 0 \\
22 & 0 \\
23 & 0 \\
24 & 0 \\
25 & 0 \\
26 & 0 \\
27 & 0 \\
28 & 0\end{array}$ & & & $\begin{array}{l}0.000 \mathrm{E}+00 \\
0.000 \mathrm{E}+00 \\
0.000 \mathrm{E}+00 \\
0.000 \mathrm{E}+00 \\
0.000 \mathrm{E}+00 \\
0.000 \mathrm{E}+00 \\
0.000 \mathrm{E}+00 \\
0.000 \mathrm{E}+00 \\
0.000 \mathrm{E}+00 \\
0.000 \mathrm{E}+00 \\
0.000 \mathrm{E}+00 \\
0.000 \mathrm{E}+00 \\
0.000 \mathrm{E}+00\end{array}$ \\
\hline
\end{tabular}


HNF-SD-WM-CN-104

Rev. 0

Page 19 of 33

\begin{tabular}{|c|c|c|c|}
\hline $\begin{array}{l}\text { Nucli } \\
\text { Group } \\
\text { No. }\end{array}$ & $\begin{array}{c}84 \text { i } \\
\text { Gamma } \\
\text { X-ray }\end{array}$ & Brems & $\begin{array}{c}(Z=39) \\
\text { Tota } 1 \\
\text { photon } / \mathrm{sec}\end{array}$ \\
\hline $\begin{array}{rl}1 & 3 . \\
2 & 0 . \\
3 & 0 . \\
4 & 0 . \\
5 & 0 . \\
6 & 0 . \\
7 & 0 . \\
8 & 0 . \\
9 & 0 . \\
10 & 0 . \\
11 & 0 . \\
12 & 0 . \\
13 & 0 . \\
14 & 0 . \\
15 & 0 .\end{array}$ & $\begin{array}{l}.009 \mathrm{E}+12 \\
.000 \mathrm{E}+00 \\
.000 \mathrm{E}+00 \\
.000 \mathrm{E}+00 \\
.000 \mathrm{E}+00 \\
.000 \mathrm{E}+00 \\
.000 \mathrm{E}+00 \\
.000 \mathrm{E}+00 \\
.000 \mathrm{E}+00 \\
.000 \mathrm{E}+00\end{array}$ & $\begin{array}{l}5.609 \\
3.497 \\
2.516 \\
1.817\end{array}$ & $\begin{array}{l}9 \mathrm{E}+14 \\
7 \mathrm{E}+14 \\
6 \mathrm{E}+14 \\
7 \mathrm{E}+14 \\
7 \mathrm{E}+14 \\
7 \mathrm{E}+14 \\
3 \mathrm{E}+14 \\
9 \mathrm{E}+14 \\
1 \mathrm{E}+14 \\
4 \mathrm{E}+14 \\
2 \mathrm{E}+13 \\
9 \mathrm{E}+13 \\
3 \mathrm{E}+13\end{array}$ \\
\hline
\end{tabular}

Weight $(84)=1.070 \mathrm{E}+06 \mathrm{Ci}$

Group Gamma Total

No. X-ray Brems photon/sec

$16 \quad 0.000 \mathrm{E}+00 \quad 1.119 \mathrm{E}+13 \quad 1.119 \mathrm{E}+13$

$170.000 \mathrm{E}+00 \quad 5.766 \mathrm{E}+12 \quad 5.766 \mathrm{E}+12$

$\begin{array}{lll}18 & 0.000 \mathrm{E}+00 & 1.908 \mathrm{E}+12 \quad 1.908 \mathrm{E}+12\end{array}$

$19 \quad 1.346 \mathrm{E}+12 \quad 4.350 \mathrm{E}+11 \quad 1.781 \mathrm{E}+12$

$200.000 \mathrm{E}+00 \quad 9.623 \mathrm{E}+10 \quad 9.623 \mathrm{E}+10$

$215.543 \mathrm{E}+10 \quad 8.072 \mathrm{E}+09 \quad 6.350 \mathrm{E}+10$

$220.000 \mathrm{E}+00 \quad 1.184 \mathrm{E}+08 \quad 1.184 \mathrm{E}+08$

$23 \quad 0.000 \mathrm{E}+00 \quad 0.000 \mathrm{E}+00 \quad 0.000 \mathrm{E}+00$

$24 \quad 0.000 \mathrm{E}+00 \quad 0.000 \mathrm{E}+00 \quad 0.000 \mathrm{E}+00$

$\begin{array}{lll}25 & 0.000 \mathrm{E}+00 \quad 0.000 \mathrm{E}+00 \quad 0.000 \mathrm{E}+00\end{array}$

$\begin{array}{lllll}26 & 0.000 \mathrm{E}+00 & 0.000 \mathrm{E}+00 & 0.000 \mathrm{E}+00\end{array}$

$27 \quad 0.000 \mathrm{E}+00 \quad 0.000 \mathrm{E}+00 \quad 0.000 \mathrm{E}+00$

$28 \quad 0.000 \mathrm{E}+00 \quad 0.000 \mathrm{E}+00 \quad 0.000 \mathrm{E}+00$

$29 \quad 0.000 E+00 \quad 0.000 E+00 \quad 0.000 E+00$

$30 \quad 0.000 \mathrm{E}+00 \quad 0.000 \mathrm{E}+00 \quad 0.000 \mathrm{E}+00$

Totals: $4.410 \mathrm{E}+12 \quad 4.087 \mathrm{E}+15 \quad 4.091 \mathrm{E}+15$

\begin{tabular}{|c|c|c|c|}
\hline $\begin{array}{l}\text { Nucl id } \\
\text { Group } \\
\text { No. }\end{array}$ & $\begin{array}{l}\text { e } 335 \text { is } \\
\text { Gamma } \\
\text { X-ray }\end{array}$ & Brems & $\begin{array}{c}(Z=55) \\
\text { Total } \\
\text { photon/sec }\end{array}$ \\
\hline $\begin{array}{rr}1 & 0 \\
2 & 0 \\
3 & 0 \\
4 & 0 \\
5 & 0 \\
6 & 0 \\
7 & 0 \\
8 & 0 \\
9 & 0 \\
10 & 0 \\
11 & 0 \\
12 & 0 \\
13 & 0 \\
14 & 0 \\
15 & 0\end{array}$ & $\begin{array}{l}00 \mathrm{E}+00 \\
00 \mathrm{E}+00 \\
00 \mathrm{E}+00 \\
00 \mathrm{E}+00 \\
00 \mathrm{E}+00 \\
00 \mathrm{E}+00 \\
00 \mathrm{E}+00 \\
00 \mathrm{E}+00 \\
00 \mathrm{E}+00 \\
00 \mathrm{E}+00\end{array}$ & $\begin{array}{l}2.641 \mathrm{E}+13 \\
1.536 \mathrm{E}+13 \\
8.182 \mathrm{E}+12 \\
4.864 \mathrm{E}+12 \\
3.670 \mathrm{E}+12 \\
2.569 \mathrm{E}+12 \\
1.974 \mathrm{E}+12 \\
1.430 \mathrm{E}+12 \\
1.091 \mathrm{E}+12 \\
4.393 \mathrm{E}+12 \\
8.532 \mathrm{E}+11 \\
2.269 \mathrm{E}+11 \\
1.031 \mathrm{E}+11\end{array}$ & $\begin{array}{l}2.641 \\
1.536 \\
8.182 \\
4.864 \\
3.670 \\
2.569 \\
1.974 \\
1.430 \\
1.091 \\
4.393 \\
8.532 \\
2.269 \\
1.031 \\
3.397\end{array}$ \\
\hline
\end{tabular}

Weight $(335)=1.620 \mathrm{E}+05 \mathrm{Ci}$ Group Gamma Total No. X-ray Brems photon/sec

$16 \quad 0.000 E+00 \quad 7.117 E+08 \quad 7.117 E+08$

$170.000 \mathrm{E}+00 \quad 5.348 \mathrm{E}+06 \quad 5.348 \mathrm{E}+06$

$18 \quad 0.000 \mathrm{E}+00 \quad 0.000 \mathrm{E}+00 \quad 0.000 \mathrm{E}+00$

$190.000 \mathrm{E}+00 \quad 0.000 \mathrm{E}+00 \quad 0.000 \mathrm{E}+00$

$20 \quad 0.000 \mathrm{E}+00 \quad 0.000 \mathrm{E}+00 \quad 0.000 \mathrm{E}+00$

$21 \quad 0.000 \mathrm{E}+00 \quad 0.000 \mathrm{E}+00 \quad 0.000 \mathrm{E}+00$

$22 \quad 0.000 \mathrm{E}+00 \quad 0.000 \mathrm{E}+00 \quad 0.000 \mathrm{E}+00$

$230.000 \mathrm{E}+00 \quad 0.000 \mathrm{E}+00 \quad 0.000 \mathrm{E}+00$

$24 \quad 0.000 \mathrm{E}+00 \quad 0.000 \mathrm{E}+00 \quad 0.000 \mathrm{E}+00$

$25 \quad 0.000 \mathrm{E}+00 \quad 0.000 \mathrm{E}+00 \quad 0.000 \mathrm{E}+00$

$\begin{array}{llll}26 & 0.000 E+00 & 0.000 E+00 & 0.000 E+00\end{array}$

$27 \quad 0.000 \mathrm{E}+00 \quad 0.000 \mathrm{E}+00 \quad 0.000 \mathrm{E}+00$

$280.000 \mathrm{E}+00 \quad 0.000 \mathrm{E}+00 \quad 0.000 \mathrm{E}+00$

$290.000 \mathrm{E}+00 \quad 0.000 \mathrm{E}+00 \quad 0.000 \mathrm{E}+00$

$30 \quad 0.000 \mathrm{E}+00 \quad 0.000 \mathrm{E}+00 \quad 0.000 \mathrm{E}+00$

Totals: $0.000 \mathrm{E}+00 \quad 7.117 \mathrm{E}+13 \quad 7.117 \mathrm{E}+13$

\begin{tabular}{|c|c|c|c|c|c|c|c|}
\hline & & & & & & & \\
\hline $\begin{array}{l}\text { Group } \\
\text { No. }\end{array}$ & $\begin{array}{l}\text { Gamma } \\
\text { X-ray }\end{array}$ & Brems & $\begin{array}{c}\text { Total } \\
\text { photon/sec }\end{array}$ & $\begin{array}{l}\text { Group } \\
\text { No. }\end{array}$ & $\begin{array}{l}\text { Gamma } \\
\text { X-ray }\end{array}$ & Brems & $\begin{array}{c}\text { Total } \\
\text { photon/sec }\end{array}$ \\
\hline $\begin{array}{rl}3 & 4.1 \\
14 & 5.0\end{array}$ & & & & & & & \\
\hline
\end{tabular}


HNF-SD-WM-CN-104

Rev. 0

Page 20 of 33

Totals: $5.506 \mathrm{E}+15 \quad 0.000 \mathrm{E}+00 \quad 5.506 \mathrm{E}+15$ 
HNF-SD-WM-CN-104

Rev. 0

Page 21 of 33

\begin{tabular}{|c|c|c|c|}
\hline $\begin{array}{l}\text { Nucl i } \\
\text { Group } \\
\text { No. }\end{array}$ & Gamma & Brems & $\begin{array}{c}(Z=63) \\
\text { Total } \\
\text { photon } / \mathrm{sec}\end{array}$ \\
\hline $\begin{array}{rl}1 & 0 \\
2 & 0 \\
3 & 0 \\
4 & 3 \\
5 & 0 \\
6 & 0 \\
7 & 0 \\
8 & 0 \\
9 & 0 \\
10 & 6 \\
11 & 1 \\
12 & 0 \\
13 & 1 \\
14 & 5 \\
15 & 2\end{array}$ & $\begin{array}{c}00 E+00 \\
00 E+00 \\
00 E+00 \\
37 E+13 \\
b 0 E+00 \\
\partial 0 E+00 \\
0 D E+00 \\
0 E+00\end{array}$ & $\begin{array}{l}9.421 \\
4.777 \\
3.120 \\
1.967 \\
1.481 \\
1.131 \\
9.334 \\
7.942 \\
6\end{array}$ & $\begin{array}{l}9.421 \mathrm{E}+11 \\
4.777 \mathrm{E}+11 \\
3.120 \mathrm{E}+11 \\
3.957 \mathrm{E}+13 \\
1.481 \mathrm{E}+11 \\
1.131 \mathrm{E}+11 \\
9.334 \mathrm{E}+10 \\
7.942 \mathrm{E}+10 \\
6.619 \mathrm{E}+10 \\
6.304 \mathrm{E}+13 \\
1.026 \mathrm{E}+13 \\
3.168 \mathrm{E}+10 \\
1.456 \mathrm{E}+12 \\
5.224 \mathrm{E}+13 \\
2.165 \mathrm{E}+13\end{array}$ \\
\hline
\end{tabular}

Weight $(415)=4.170 \mathrm{E}+03 \mathrm{Ci}$

Group Gamma Total

No. X-ray Brems photon/sec

$164.369 \mathrm{E}+13 \quad 1.765 \mathrm{E}+09 \quad 4.370 \mathrm{E}+13$

$17 \quad 5.726 \mathrm{E}+13 \quad 6.375 \mathrm{E}+08 \quad 5.726 \mathrm{E}+13$

$18 \quad 5.446 \mathrm{E}+12 \quad 1.013 \mathrm{E}+08 \quad 5.447 \mathrm{E}+12$

$190.000 \mathrm{E}+00 \quad 4.246 \mathrm{E}+06 \quad 4.246 \mathrm{E}+06$

$200.000 E+00 \quad 4.041 E+03 \quad 4.041 E+03$

$210.000 \mathrm{E}+00 \quad 0.000 \mathrm{E}+00 \quad 0.000 \mathrm{E}+00$

$22 \quad 0.000 \mathrm{E}+00 \quad 0.000 \mathrm{E}+00 \quad 0.000 \mathrm{E}+00$

$23 \quad 0.000 \mathrm{E}+00 \quad 0.000 \mathrm{E}+00 \quad 0.000 \mathrm{E}+00$

$24 \quad 0.000 E+00 \quad 0.000 E+00 \quad 0.000 E+00$

$25 \quad 0.000 \mathrm{E}+00 \quad 0.000 \mathrm{E}+00 \quad 0.000 \mathrm{E}+00$

$26 \quad 0.000 \mathrm{E}+00 \quad 0.000 \mathrm{E}+00 \quad 0.000 \mathrm{E}+00$

$27 \quad 0.000 \mathrm{E}+00 \quad 0.000 \mathrm{E}+00 \quad 0.000 \mathrm{E}+00$

$\begin{array}{llll}28 & 0.000 E+00 & 0.000 E+00 & 0.000 E+00\end{array}$

$290.000 E+00 \quad 0.000 E+00 \quad 0.000 E+00$

$\begin{array}{ll}30 & 0.000 \mathrm{E}+00 \quad 0.000 \mathrm{E}+00 \quad 0.000 \mathrm{E}+00\end{array}$

Totals: $2.940 \mathrm{E}+14 \quad 2.837 \mathrm{E}+12 \quad 2.969 \mathrm{E}+14$

\begin{tabular}{|c|c|c|c|}
\hline $\begin{array}{l}\text { Nucl i } \\
\text { Group } \\
\text { No. }\end{array}$ & $\underset{\substack{\text { Gamma } \\
\text { X-ray }}}{502 \text { is }}$ & $\begin{array}{r}\text { s NP-237 } \\
\text { Brems }\end{array}$ & $\begin{array}{c}(Z=93) \\
\text { Total } \\
\text { photon } / \mathrm{sec}\end{array}$ \\
\hline $\begin{array}{rr}1 & 1 \\
2 & 4 \\
3 & 0 \\
4 & 4 \\
5 & 1 \\
6 & 0 \\
7 & 0 \\
8 & 3 \\
9 & 1 \\
10 & 9 \\
11 & 4\end{array}$ & $\begin{array}{l}595 E+12 \\
006 E+11 \\
000 E+00 \\
009 E+09 \\
203 E+10 \\
000 E+00 \\
000 E+00 \\
554 E+11 \\
32 E+11 \\
151 E+10 \\
582 E+09\end{array}$ & $\begin{array}{l}1.693 \mathrm{E}+08 \\
2.967 \mathrm{E}+07 \\
6.926 \mathrm{E}+06 \\
1.857 \mathrm{E}+06 \\
5.607 \mathrm{E}+05 \\
1.823 \mathrm{E}+05 \\
6.112 \mathrm{E}+04 \\
1.978 \mathrm{E}+04 \\
4.878 \mathrm{E}+03 \\
1.909 \mathrm{E}+03 \\
0.000 \mathrm{E}+00\end{array}$ & $\begin{array}{l}1.695 \mathrm{E}+12 \\
4.007 \mathrm{E}+11 \\
6.926 \mathrm{E}+06 \\
4.011 \mathrm{E}+09 \\
1.203 \mathrm{E}+10 \\
1.823 \mathrm{E}+05 \\
6.112 \mathrm{E}+04 \\
3.654 \mathrm{E}+11 \\
1.432 \mathrm{E}+11 \\
9.451 \mathrm{E}+10 \\
4.582 \mathrm{E}+09\end{array}$ \\
\hline
\end{tabular}

Weight $(502)=7.740 \mathrm{E}+01 \mathrm{Ci}$

Group Gamma Total

No. X-ray Brems photon/sec

$\begin{array}{llll}16 & 0.000 \mathrm{E}+00 & 0.000 \mathrm{E}+00 & 0.000 \mathrm{E}+00\end{array}$

$17 \quad 0.000 \mathrm{E}+00 \quad 0.000 \mathrm{E}+00 \quad 0.000 \mathrm{E}+00$

$18 \quad 0.000 \mathrm{E}+00 \quad 0.000 \mathrm{E}+00 \quad 0.000 \mathrm{E}+00$

$19 \quad 0.000 \mathrm{E}+00 \quad 0.000 \mathrm{E}+00 \quad 0.000 \mathrm{E}+00$

$20 \quad 0.000 \mathrm{E}+00 \quad 0.000 \mathrm{E}+00 \quad 0.000 \mathrm{E}+00$

$21 \quad 0.000 \mathrm{E}+00 \quad 0.000 \mathrm{E}+00 \quad 0.000 \mathrm{E}+00$

$22 \quad 0.000 \mathrm{E}+00 \quad 0.000 \mathrm{E}+00 \quad 0.000 \mathrm{E}+00$

$23 \quad 0.000 \mathrm{E}+00 \quad 0.000 \mathrm{E}+00 \quad 0.000 \mathrm{E}+00$

$240.000 \mathrm{E}+00 \quad 0.000 \mathrm{E}+00 \quad 0.000 \mathrm{E}+00$

$25 \quad 0.000 \mathrm{E}+00 \quad 0.000 \mathrm{E}+00 \quad 0.000 \mathrm{E}+00$

$\begin{array}{llll}26 & 0.000 \mathrm{E}+00 & 0.000 \mathrm{E}+00 & 0.000 \mathrm{E}+00\end{array}$

Totals: $2.719 \mathrm{E}+12 \quad 2.086 \mathrm{E}+08 \quad 2.720 \mathrm{E}+12$

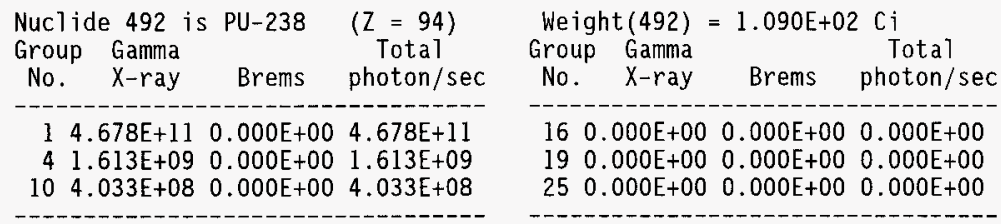

Totals: $4.698 \mathrm{E}+11 \quad 0.000 \mathrm{E}+00 \quad 4.698 \mathrm{E}+11$ 
HNF-SD-WM-CN-104

Rev. 0

Page 22 of 33 


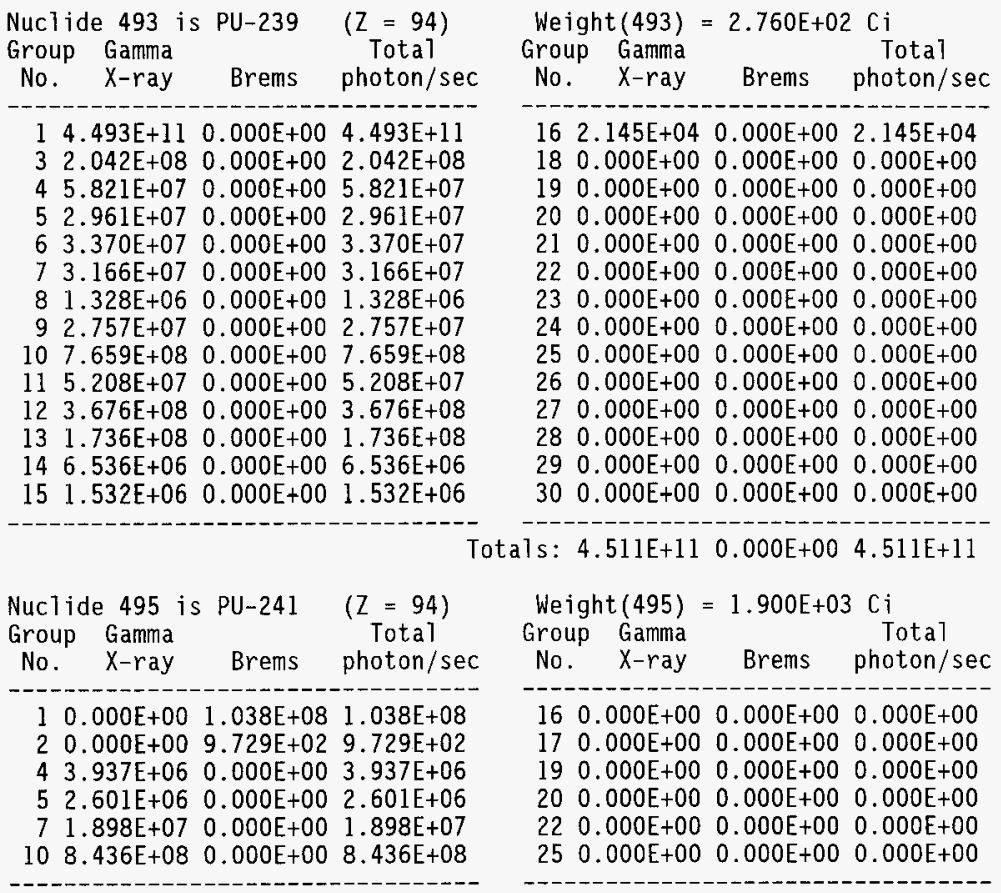

Totals: $8.691 \mathrm{E}+08 \quad 1.038 \mathrm{E}+08 \quad 9.730 \mathrm{E}+08$ 
HNF-SD-WM-CN-104

Rev. 0

Page 24 of 33

\begin{tabular}{|c|c|c|c|c|c|c|c|}
\hline $\begin{array}{l}\text { Nuclide } \\
\text { Group } \\
\text { No. }\end{array}$ & $\begin{array}{l}496 \text { is } \\
\text { Gamma } \\
x-\text { ray }\end{array}$ & AM-241 & $\begin{array}{c}(Z=95) \\
\text { Total } \\
\text { photon/sec }\end{array}$ & $\begin{array}{l}\text { Weight } \\
\text { Group } \\
\text { No. }\end{array}$ & $\begin{array}{l}(496)= \\
\text { Gamma } \\
x-r a y\end{array}$ & $\begin{array}{c}8.080 E+02 \\
\text { Brems }\end{array}$ & $\begin{array}{l}\mathrm{Ci} \\
\text { Total } \\
\text { photon/sec }\end{array}$ \\
\hline $\begin{array}{rl}1 & 1.2 \\
2 & 7.1 \\
3 & 3.2 \\
4 & 2.6 \\
5 & 1.0 \\
6 & 5.9 \\
7 & 0.0 \\
8 & 0.0 \\
9 & 6.2 \\
10 & 8.3 \\
11 & 2.5 \\
12 & 3.8 \\
13 & 2.3 \\
14 & 2.0 \\
15 & 6.5\end{array}$ & $\begin{array}{l}86 \mathrm{E}+13 \\
775 \mathrm{E}+11 \\
899 \mathrm{E}+10 \\
91 \mathrm{E}+10 \\
67 \mathrm{E}+13 \\
379 \mathrm{E}+08 \\
00 \mathrm{E}+00 \\
300 \mathrm{E}+00 \\
778 \mathrm{E}+09 \\
371 \mathrm{E}+09 \\
571 \mathrm{E}+08 \\
386 \mathrm{E}+08 \\
332 \mathrm{E}+07 \\
63 \mathrm{E}+08 \\
577 \mathrm{E}+06\end{array}$ & $\begin{array}{l}5.000 \mathrm{E}+08 \\
5.192 \mathrm{E}+07 \\
6.120 \mathrm{E}+06 \\
6.691 \mathrm{E}+05 \\
1.018 \mathrm{E}+05 \\
1.929 \mathrm{E}+04 \\
2.758 \mathrm{E}+03 \\
1.834 \mathrm{E}+02 \\
1.298 \mathrm{E}+00 \\
0.000 \mathrm{E}+00 \\
0.000 \mathrm{E}+00 \\
0.000 \mathrm{E}+00 \\
0.000 \mathrm{E}+00 \\
0.000 \mathrm{E}+00 \\
0.000 \mathrm{E}+00\end{array}$ & $\begin{array}{l}1.286 \mathrm{E}+13 \\
7.176 \mathrm{E}+11 \\
3.289 \mathrm{E}+10 \\
2.691 \mathrm{E}+10 \\
1.067 \mathrm{E}+13 \\
5.979 \mathrm{E}+08 \\
2.758 \mathrm{E}+03 \\
1.834 \mathrm{E}+02 \\
6.278 \mathrm{E}+09 \\
8.371 \mathrm{E}+09 \\
2.571 \mathrm{E}+08 \\
3.886 \mathrm{E}+08 \\
2.332 \mathrm{E}+07 \\
2.063 \mathrm{E}+08 \\
6.577 \mathrm{E}+06\end{array}$ & $\begin{array}{ll}16 & 0 . \\
17 & 0 . \\
18 & 0 . \\
19 & 0 . \\
20 & 0 . \\
21 & 0 . \\
22 & 0 . \\
23 & 0 . \\
24 & 0 . \\
25 & 0 . \\
25 & 0 . \\
27 & 0 . \\
28 & 0 . \\
29 & 0 . \\
30 & 0 .\end{array}$ & $\begin{array}{l}000 E+00 \\
000 E+00 \\
000 E+00 \\
000 E+00 \\
000 E+00 \\
000 E+00 \\
000 E+00 \\
000 E+00 \\
000 E+00 \\
000 E+00 \\
000 E+00 \\
000 E+00 \\
000 E+00 \\
000 E+00 \\
000 E+00\end{array}$ & $\begin{array}{l}0.000 E+00 \\
0.000 E+00 \\
0.000 E+00 \\
0.000 E+00 \\
0.000 E+00 \\
0.000 E+00 \\
0.000 E+00 \\
0.000 E+00 \\
0.000 E+00 \\
0.000 E+00 \\
0.000 E+00 \\
0.000 E+00 \\
0.000 E+00 \\
0.000 E+00 \\
0.000 E+00\end{array}$ & $\begin{array}{l}0.000 \mathrm{E}+00 \\
0.000 \mathrm{E}+00 \\
0.000 \mathrm{E}+00 \\
0.000 \mathrm{E}+00 \\
0.000 \mathrm{E}+00 \\
0.000 \mathrm{E}+00 \\
0.000 \mathrm{E}+00 \\
0.000 \mathrm{E}+00 \\
0.000 \mathrm{E}+00 \\
0.000 \mathrm{E}+00 \\
0.000 \mathrm{E}+00 \\
0.000 \mathrm{E}+00 \\
0.000 \mathrm{E}+00 \\
0.000 \mathrm{E}+00 \\
0.000 \mathrm{E}+00\end{array}$ \\
\hline \multicolumn{8}{|c|}{ Totals: $2.432 \mathrm{E}+13 \quad 5.588 \mathrm{E}+08 \quad 2.432 \mathrm{E}+13$} \\
\hline $\begin{array}{l}\text { Nuclide } \\
\text { Group } \\
\text { No. }\end{array}$ & $\begin{array}{l}500 \text { is } \\
\text { Gamma } \\
\text { X-ray }\end{array}$ & $\begin{array}{l}\text { CM-244 } \\
\text { Brems }\end{array}$ & $\begin{array}{c}(Z=96) \\
\text { Total } \\
\text { photon } / \mathrm{sec}\end{array}$ & $\begin{array}{l}\text { Weigh } \\
\text { Group } \\
\text { No. }\end{array}$ & $\begin{array}{r}t(500)= \\
\text { Gamma } \\
x-r a y\end{array}$ & $\begin{array}{c}=5.080 \mathrm{E}+00 \\
\text { Brems }\end{array}$ & $\begin{array}{l}\text { C } \mathrm{Ci} \\
\text { Total } \\
\text { photon/sec }\end{array}$ \\
\hline $\begin{array}{ll}1 & 3.3 \\
4 & 1.6\end{array}$ & $3 \mathrm{E}+10$ & $0.000 E+00$ & 3. $383 \mathrm{E}+10$ & $\begin{array}{ll}16 & 0 \\
19 & 0\end{array}$ & $\begin{array}{l}000 E+00 \\
000 E+00\end{array}$ & $\begin{array}{l}0.000 E+00 \\
0.000 E+00\end{array}$ & $00 E+00$ \\
\hline
\end{tabular}

Totals: $3.400 \mathrm{E}+10 \quad 0.000 \mathrm{E}+00 \quad 3.400 \mathrm{E}+10$ 
HNF-SD-WM-CN-104

Rev. 0

Page 25 of 33

MITIGATED 12 HOUR RELEASE - $100 \mathrm{M}$ AWAY

$\begin{array}{ccl}\text { Source } & \text { Shields } & \text { Distance to Detector, } X=1.023 \mathrm{E}+04 \mathrm{~cm} \\ \text { Slab } & \text { Slab } & \text { Source Volume }=1.703 \mathrm{E}+08 \mathrm{cc} \\ & \text { Source Mass }=3.677 \mathrm{E}+08 \mathrm{grams}\end{array}$

Thickness $=2.134 \mathrm{E}+02 \mathrm{~cm} \quad$ Height $=6.096 \mathrm{E}+01 \mathrm{~cm} \quad$ Width $=1.309 \mathrm{E}+04 \mathrm{~cm}$ Integration Specs: NTHETA $=15 \quad$ NPSI $=15 \quad D E L R=5.080 \mathrm{E}+00 \mathrm{~cm}$

Total Intervals: $9.450 E+03$ (photon source is the lst region)

Shield Thickness: $2.134 \mathrm{E}+02,1.524 \mathrm{E}+01,1.000 \mathrm{E}+04 \mathrm{~cm}$

Distances from Dose Point to the Outside of

(1) Source Region: $1.002 E+04 \mathrm{~cm}$ (2) Next Layer: $1.000 \mathrm{E}+04 \mathrm{~cm}$

Dose Buildup Data for Shield 2 with Effective Atomic Number 10.78

Fluence-to-Dose Conversion: Photons in Air

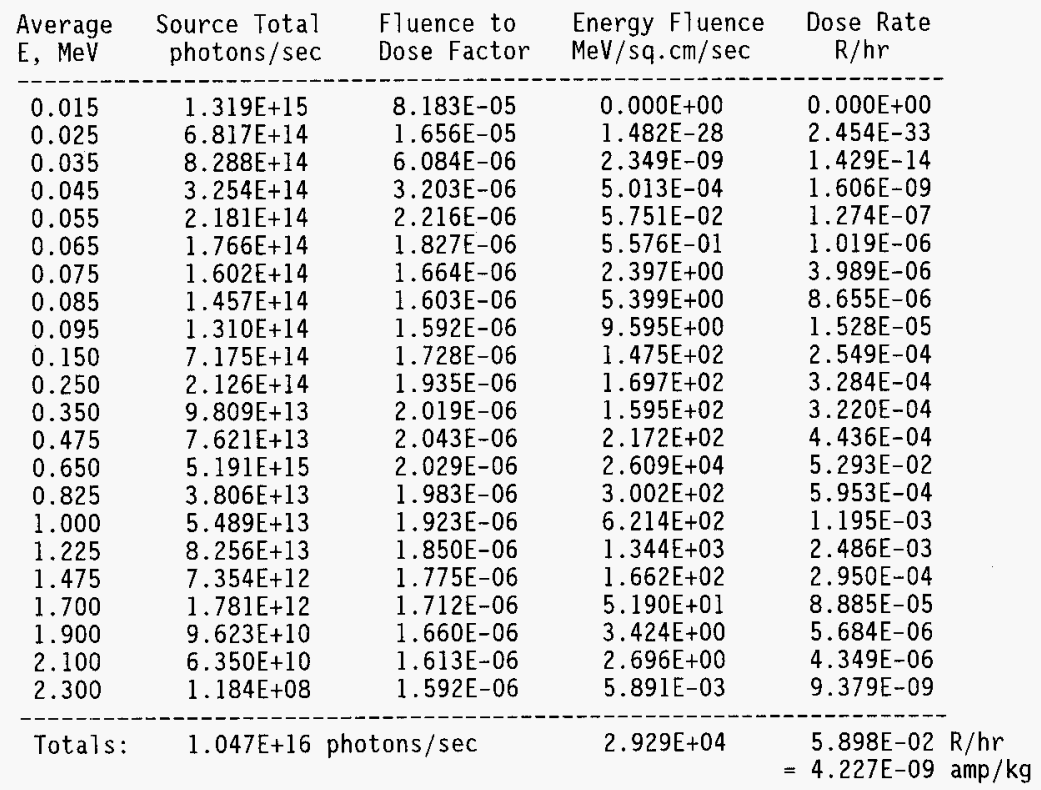


HNF-SD-WM-CN-104

Rev. 0

Page 26 of 33

\begin{tabular}{|c|c|c|c|c|c|c|}
\hline $\begin{array}{c}\text { erage } \\
\mathrm{Mev}\end{array}$ & $N I T=1$ & $\begin{array}{l}\text { Posterior } \\
\text { DUNIT = } 2\end{array}$ & $\begin{array}{c}\text { Lateral } \\
\text { DUNIT }=3\end{array}$ & $\begin{array}{l}\text { Rotational } \\
\text { DUNIT }=4\end{array}$ & $\begin{array}{l}\text { Isometric } \\
\text { DUNIT }=5\end{array}$ & $\begin{array}{l}\text { Orig ANSI } \\
\text { DUNIT }=6\end{array}$ \\
\hline $\begin{array}{l}0.025 \\
0.035 \\
0.045 \\
0.055 \\
0.065 \\
0.075 \\
0.085 \\
0.095 \\
0.150 \\
0.250 \\
0.350 \\
0.475 \\
0.650 \\
0.825 \\
1.000 \\
1.225 \\
1.475 \\
1.700 \\
1.900 \\
2.100 \\
2.300\end{array}$ & $\begin{array}{l}6.145 \mathrm{E}-34 \\
8.264 \mathrm{E}-15 \\
1.493 \mathrm{E}-09 \\
1.494 \mathrm{E}-07 \\
1.303 \mathrm{E}-06 \\
5.165 \mathrm{E}-06 \\
1.095 \mathrm{E}-05 \\
1.861 \mathrm{E}-05 \\
2.656 \mathrm{E}-04 \\
3.174 \mathrm{E}-04 \\
2.983 \mathrm{E}-04 \\
3.991 \mathrm{E}-04 \\
4.630 \mathrm{E}-02 \\
5.131 \mathrm{E}-04 \\
1.024 \mathrm{E}-03 \\
2.117 \mathrm{E}-03 \\
2.501 \mathrm{E}-04\end{array}$ & $\begin{array}{l}2.810 \mathrm{E}-34 \\
4.785 \mathrm{E}-15 \\
9.544 \mathrm{E}-10 \\
1.012 \mathrm{E}-07 \\
9.213 \mathrm{E}-07 \\
3.786 \mathrm{E}-06 \\
8.281 \mathrm{E}-06 \\
1.446 \mathrm{E}-05 \\
2.218 \mathrm{E}-04 \\
2.625 \mathrm{E}-04 \\
2.508 \mathrm{E}-04 \\
3.430 \mathrm{E}-04 \\
4.080 \mathrm{E}-02 \\
4.607 \mathrm{E}-04 \\
9.319 \mathrm{E}-04 \\
1.952 \mathrm{E}-03 \\
2.330 \mathrm{E}-04 \\
7.055 \mathrm{E}-05 \\
4.534 \mathrm{E}-06 \\
3.482 \mathrm{E}-06 \\
7.429 \mathrm{E}-09\end{array}$ & & $\begin{array}{l}2.989 \mathrm{E}-34 \\
4.391 \mathrm{E}-15 \\
8.451 \mathrm{E}-10 \\
8.845 \mathrm{E}-08 \\
7.970 \mathrm{E}-07 \\
3.241 \mathrm{E}-06 \\
7.018 \mathrm{E}-06 \\
1.215 \mathrm{E}-05 \\
1.903 \mathrm{E}-04 \\
2.286 \mathrm{E}-04 \\
2.205 \mathrm{E}-04 \\
3.040 \mathrm{E}-04 \\
3.644 \mathrm{E}-02 \\
4.134 \mathrm{E}-04 \\
8.391 \mathrm{E}-04 \\
1.763 \mathrm{E}-03 \\
2.110 \mathrm{E}-04 \\
6.398 \mathrm{E}-05 \\
4.117 \mathrm{E}-06 \\
3.165 \mathrm{E}-06 \\
6.759 \mathrm{E}-09\end{array}$ & & $\begin{array}{l}4.742 \mathrm{E}-33 \\
2.938 \mathrm{E}-14 \\
3.532 \mathrm{E}-09 \\
2.867 \mathrm{E}-07 \\
2.226 \mathrm{E}-06 \\
8.255 \mathrm{E}-06 \\
1.681 \mathrm{E}-05 \\
2.785 \mathrm{E}-05 \\
3.730 \mathrm{E}-04 \\
4.283 \mathrm{E}-04 \\
4.002 \mathrm{E}-04 \\
5.105 \mathrm{E}-04 \\
5.787 \mathrm{E}-02 \\
6.262 \mathrm{E}-04 \\
1.230 \mathrm{E}-03 \\
2.509 \mathrm{E}-03 \\
2.935 \mathrm{E}-04 \\
8.769 \mathrm{E}-05 \\
5.584 \mathrm{E}-06 \\
4.256 \mathrm{E}-06 \\
9.021 \mathrm{E}-09\end{array}$ \\
\hline & & & & $.0 / U E-U L$ & $3.479 \mathrm{E}-02$ & $6.440 \mathrm{E}-0$ \\
\hline
\end{tabular}

Closing: DAT'S ALL PHOLQUES!!!!!!!!

Finish run at 13:57:49 05/05/97 
Input File (D:\ISOSHLD INPUT\BIRMLEAK.) is shown below:

0 UNMITIGATED 12 HOUR RELEASE - $100 \mathrm{M}$ AWAY

\&INPUT NEXT $=1$, IGEOM=10, NTHETA=15, NPSI $=15, D E L R=5.0$, $T(1)=213.36, T(2)=15.24, X=10228.6$, SL $T H=13089.99, Y=60.96$, IPRNT $=0$, OPTION=1, ISPEC $=3, N S H L D=2, J B U F=2$, DUNIT $=7$, WEIGHT $(472)=3.02 E+2$, WEIGHT (082) $=1.79 \mathrm{E}+6$, WEIGHT (084) $=1.79 \mathrm{E}+6$, WEIGHT $(335)=1.69 \mathrm{E}+5$, WEIGHT $(336)=1.60 \mathrm{E}+5$, WEIGHT (415) $=6.75 \mathrm{E}+3$, WEIGHT (502) $=6.08 \mathrm{E}+2$, WEIGHT $(492)=4.11 \mathrm{E}+1$, WEIGHT (493) $=2.71 \mathrm{E}+2$, \& WEIGHT $(495)=1.04 \mathrm{E}+3$, WEIGHT $(496)=6.75 \mathrm{E}+3$, WEIGHT $(500)=3.74 \mathrm{E}+1$,
SOIL 211.6
1.6

1 WATER 10.56

MITIGATED 12 HOUR RELEASE - $100 \mathrm{M}$ AWAY

\&INPUT NEXT $=1$, IGEOM $=10$, NTHETA=15, NPSI $=15, D E L R=5.0$, $T(1)=213.36, T(2)=15.24, X=10228.6, S L T H=13089.99, Y=60.96$, IPRNT $=0$, OPTION=1, ISPEC $=3, \mathrm{NSHLD}=2, \mathrm{JBUF}=2$, DUNIT $=7$, WEIGHT $(472)=2.64 \mathrm{E}+2$, WEIGHT $(082)=1.07 \mathrm{E}+6$, WEIGHT $(084)=1.07 \mathrm{E}+6$, WEIGHT $(335)=1.62 \mathrm{E}+5$, WEIGHT $(336)=1.53 \mathrm{E}+5$, WEIGHT $(415)=4.17 \mathrm{E}+3$, WEIGHT $(502)=7.74 \mathrm{E}+1$, WEIGHT (492) $=1.09 \mathrm{E}+2$, WEIGHT (493) $=2.76 \mathrm{E}+2$, \& WEIGHT $(495)=1.90 \mathrm{E}+3$, WEIGHT $(496)=8.08 \mathrm{E}+2$, WEIGHT $(500)=5.08 \mathrm{E}+0$, SOIL $211.6 \quad 1.6$ 1 WATER 10.56

DAT'S ALL PHOLQUES!!!!!!!! \&INPUT NEXT $=6$, \& 
HNF-SD-WM-CN-104

Rev. 0

Page 28 of 33

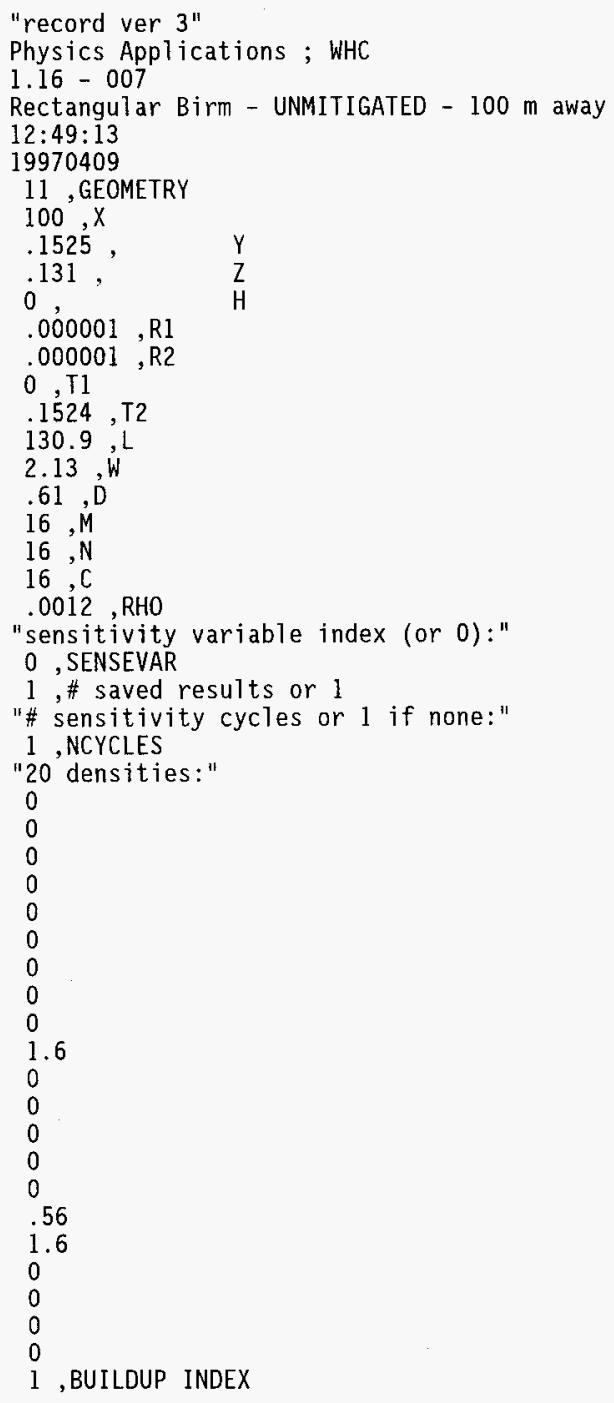


16 ,QUADRATURE ORDER

"20 energies:"

2

1.7

1.475

1.225

1

.825

.65

.475

.35

.25

.15

0

0

0

0

0

0

0

0

0

" 20 activities:"

267200000000

$2.98 \mathrm{e}+12$

1. $201 \mathrm{e}+13$

1. $247 \mathrm{e}+14$

$8.945 \mathrm{e}+13$

$6.249 e+13$

$5.487 e+15$

1. $273 \mathrm{e}+14$

$1.64 \mathrm{e}+14$

3. $547 \mathrm{e}+14$

1. $195 \mathrm{e}+15$

0

0

0

0

0

0

0

0

0

$1,1=$ RESULTS EXIST $0=$ NO

"20 results - reference (or single) case:"

$1.39225 \mathrm{e}-21$

$1.27566 \mathrm{e}-21$

$1.16583 \mathrm{e}-21$

$1.24592 \mathrm{e}-21$

$1.12573 e-21$ 
HNF-SD-WM-CN-104

Rev. 0

Page 30 of 33

\section{$9.59863 \mathrm{e}-22$}

$9.12636 \mathrm{e}-22$

$7.84988 \mathrm{e}-22$

$5.91555 \mathrm{e}-22$

$4.68345 \mathrm{e}-22$

$2.44705 \mathrm{e}-22$

0

0

0

0

0

0

0

0

0

"end of results."

3 , SOURCE MODE

"isotope bit flags:"

ब्व

4 , NUMBER OF ISOTOPES

"4 source inputs in curies:" 319000

603

337000

13500

"end of source inputs. 5 decay times:" 0

0

0

0

0 
HNF-SD-WM-CN-104

Rev. 0

Page 31 of 33

"record ver 3"

Physics Applications ; WHC

$1.16-007$

Rectangular Birm - MITIGATED - 100 m away

$14: 41: 28$

19970409

11 , GEOMETRY

$100, X$

.1525 ,

.131 ,

Y

0 ,

$\mathrm{H}$

$.000001, \mathrm{R} 1$

$.000001, R 2$

$0, \mathrm{~T} 1$

$.1524, \mathrm{~T} 2$

$130.9, \mathrm{~L}$

$2.13, W$

$.61, \mathrm{D}$

$16, M$

$16, \mathrm{~N}$

$16, \mathrm{C}$

.0012 , RHO

"sensitivity variable index (or 0):" 0 , SENSEVAR

1 , \# saved results or 1

"\# sensitivity cycles or 1 if none:"

1 , NCYCLES

"20 densities:"

0

0

0

0

0

0

0

0

1.6

0

0

0

0

0

.56

1.6

0

0

0

1 , BUILDUP INDEX 
HNF-SD-WM-CN-104

Rev. 0

Page 32 of 33

16 ,QUADRATURE ORDER

"20 energies:"

2

1.7

1.475

1.225

1

.825

.65

.475

.35

.25

.15

0

0

0

0

0

0

0

0

0

"20 activities:"

159730000000

$1.781 \mathrm{e}+12$

$7.354 e+12$

8. $256 \mathrm{e}+13$

$5.489 \mathrm{e}+13$

$3.806 e+13$

$5.191 e+15$

$7.621 \mathrm{e}+13$

$9.809 \mathrm{e}+13$

2. $126 \mathrm{e}+14$

$7.175 \mathrm{e}+14$

0

0

0

0

0

0

0

0

0

$1,1=$ RESULTS EXIST $0=N 0$

"20 results - reference (or single) case:" $1.39225 \mathrm{e}-21$

$1.27566 \mathrm{e}-21$

$1.16583 \mathrm{e}-21$

1. $24592 \mathrm{e}-21$

1. $12573 \mathrm{e}-21$ 


$$
\begin{array}{r}
\text { HNF-SD-WM-CN-104 } \\
\text { Rev. } 0 \\
\text { Page } 33 \text { of } 33
\end{array}
$$

\section{$9.59863 \mathrm{e}-22$}

$9.12636 \mathrm{e}-22$

$7.84988 \mathrm{e}-22$

$5.91555 \mathrm{e}-22$

$4.68345 \mathrm{e}-22$

2. $44705 \mathrm{e}-22$

0

0

0

0

0

0

0

0

0

"end of results."

3 , SOURCE MODE

"isotope bit flags:" (1)

4 , NUMBER OF ISOTOPES

"4 source inputs in curies:" 319000

603

337000

13500

"end of source inputs. 5 decay times:"

0

0

0

0

0 


\section{DISTRIBUTION SHEET}

\begin{tabular}{|c|c|c|c|c|c|}
\hline \multirow{2}{*}{$\begin{array}{l}\text { To } \\
\text { Distribution }\end{array}$} & \multirow{2}{*}{\multicolumn{3}{|c|}{$\begin{array}{l}\text { From } \\
\text { Criticality and Shielding }\end{array}$}} & \multicolumn{2}{|l|}{ Page 1 of 1} \\
\hline & & & & \multicolumn{2}{|c|}{ Date May 12, 1997} \\
\hline \multicolumn{4}{|c|}{ Project Title/Work Order } & \multicolumn{2}{|c|}{ EDT No. 621286} \\
\hline \multicolumn{4}{|c|}{$\begin{array}{l}\text { Calculation Note for an Underground Leak which Remains } \\
\text { Underground }\end{array}$} & \multicolumn{2}{|l|}{ ECN No. } \\
\hline Name & MSIN & $\begin{array}{c}\text { Text } \\
\text { With All } \\
\text { Attach. }\end{array}$ & Text Only & $\begin{array}{l}\text { Attach./ } \\
\text { Appendix } \\
\text { Only }\end{array}$ & $\begin{array}{l}\text { EDT/ECN } \\
\text { Only }\end{array}$ \\
\hline
\end{tabular}

J. C. Conner

A2-25 $X$

H. J. Goldberg

HO-35 X

J. Greenborg

HO-35 $X$

J. P. Harris

S2-48 $\quad x$

B. E. Hey

A3-34 $X$

R. H. Ruben

HO-35 X

R. G. Stickney

R1-49 X

R. J. Van Vleet

A3-34 $X$

Project Files

R1-29 X

Docket Files (2)

B1-17 X

TWRS S\&L Files (2)

A2-26 X

Central Files (1 + Original)

A3-88 $X$

